\title{
Toward Verification of USM3D Extensions for Mixed Element Grids
}

\author{
Mohagna J. Pandya ${ }^{1}$ and Neal T. Frink ${ }^{2}$ \\ NASA Langley Research Center, Hampton, Virginia 23681, USA \\ Ejiang Ding ${ }^{3}$ \\ Analytical Services and Materials, Inc., Hampton, Virginia 23666, USA \\ and \\ Edward B. Parlette \\ ViGYAN, Hampton, Virginia 23666, USA
}

The unstructured tetrahedral grid cell-centered finite volume flow solver USM3D has been recently extended to handle mixed element grids composed of hexahedral, prismatic, pyramidal, and tetrahedral cells. Presently, two turbulence models, namely, baseline Spalart-Allmaras (SA) and Menter Shear Stress Transport (SST), support mixed element grids. This paper provides an overview of the various numerical discretization options available in the newly enhanced USM3D. Using the SA model, the flow solver extensions are verified on three two-dimensional test cases available on the Turbulence Modeling Resource website at the NASA Langley Research Center. The test cases are zero pressure gradient flat plate, planar shear, and bump-in-channel. The effect of cell topologies on the flow solution is also investigated using the planar shear case. Finally, the assessment of various cell and face gradient options is performed on the zero pressure gradient flat plate case.

\section{Nomenclature}

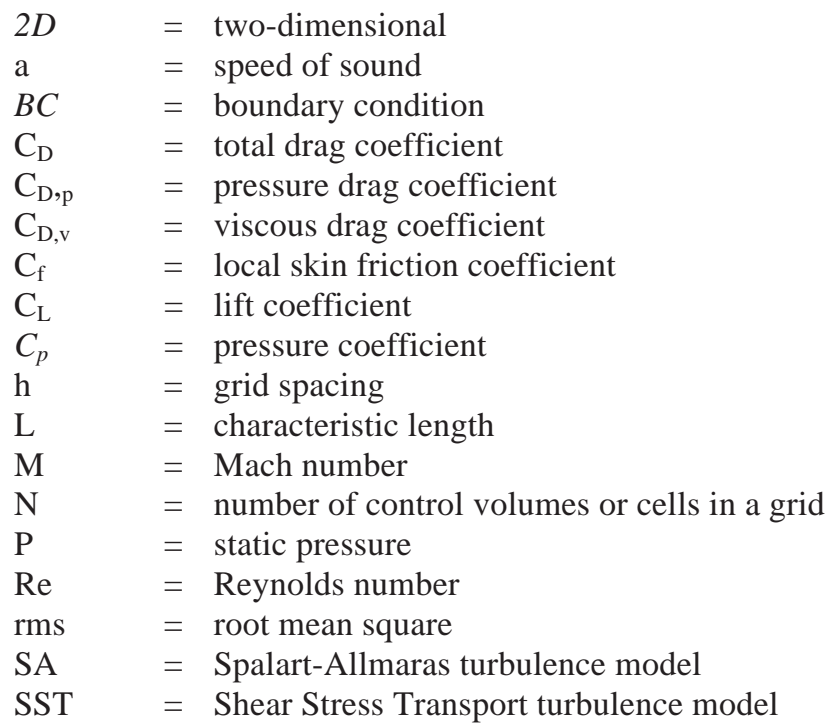

\footnotetext{
${ }^{1}$ Research Aerospace Engineer, Configuration Aerodynamics Branch, Mail Stop 499, Senior Member AIAA.

${ }^{2}$ Senior Aerospace Engineer, Configuration Aerodynamics Branch, Mail Stop 499, Associate Fellow AIAA.

${ }^{3}$ Senior Scientist, 107 Research Drive.

${ }^{4}$ Research Engineer, 30 Research Drive.
} 


$\begin{array}{ll}\mathrm{T} & =\text { static temperature } \\ \mathrm{U} & =\text { velocity magnitude } \\ \mathrm{u} & =\text { streamwise velocity } \\ \mathrm{u}+ & =\text { inner wall variable for streamwise velocity } \\ X & =\text { streamwise coordinate direction } \\ \mathrm{y}+ & =\text { inner wall variable for distance } \\ \mathrm{Z} & =\text { coordinate direction normal to the aerodynamic body } \\ \mu_{\mathrm{t}} & =\text { eddy viscosity } \\ \mu & =\text { laminar viscosity } \\ \text { Subscripts } & \\ \text { ref } & =\text { reference condition } \\ t & =\text { stagnation condition } \\ \infty & =\text { freestream condition }\end{array}$

\section{Introduction}

The NASA Tetrahedral Unstructured Software System (TetrUSS) [1,2] was developed during the 1990s to provide a rapid aerodynamic analysis and design capability to applied aerodynamicists. The system is comprised of loosely integrated, user-friendly software that enables the application of advanced Euler and Navier-Stokes tetrahedral finite volume technology to complex aerodynamic problems. The system consists of component software for setting up geometric surface definitions (GridTool) [3], generating tetrahedral grids (VGRID) [4-6], computing Euler and Navier-Stokes flow solutions (USM3D) [7-12], and extracting meaningful information from analysis of results (SimpleView). The system is also coupled with the design tool CDISC [13]. Significant extensions in VGRID and USM3D capability are described in Ref. [2].

The USM3D flow solver has been widely used over the last two decades within NASA [14, 15], U.S. government [16] and industry [17] as a workhorse for aerodynamic analysis of complex configurations. A recent example is its role as a lead CFD flow solver within the Ares project of the NASA Constellation Program, furnishing over 7,000 solutions for the vehicles up through supersonic speeds [14,15]. USM3D was utilized in the aerodynamic development of the Crew Exploration Vehicle and its Launch Abort System configuration, providing over 1,000 solutions [2]. USM3D is also being used extensively in the NASA Aviation Safety Program to generate dynamic stability and control databases for civil transports for stall/post-stall flight regimes [18].

Some of the speed of USM3D computations is attributed to an analytical formulation of the spatial differencing stencil that exploits invariant features of tetrahedra $[8,19]$, which renders it unnecessary to explicitly compute and store the cell gradients. This advantage is complimented by the VGRID tetrahedral grid generator, which generates organized thin layers of tetrahedral cells in the near-wall boundary layer region using Advancing Layer Method (ALM) [5], as illustrated in Fig. 1. Unfortunately, the diagonal faces and skewed topology of the highly stretched tetrahedra result in a severely distorted spatial differencing stencil for computing fluxes, which increases the numerical dissipation within the near-wall flow solution. Other cell topologies, such as hexahedra and prisms do not manifest this property.

Since many recent applications have ventured into predominately separated flow regimes, attention is warranted on the sensitivity of smooth surface flow separation, such as a wing leading edge near stall, to near-wall cell topology. A similar case can be made for computing heat transfer on high-speed bodies. The accuracy of USM3D simulations for such flows can be improved by using more flow-aligned hexahedral or prismatic cells in the boundary layer that transition to tetrahedral cells away from the surfaces.

Work is underway to expand the available cell topologies within USM3D to include hexahedra, prisms, and transitional pyramids. This paper describes those extensions and presents results from an initial code verification study. This study utilizes three two-dimensional test cases available on the Turbulence Modeling Resource website at the NASA Langley Research Center; the zero pressure gradient flat plate, planar shear, and bump-in-channel. Code verification is first performed on hexahedral grids for direct comparison to solutions from a well-established structured-grid code. The effect of cell topologies on the flow solution is assessed using a planar shear case. Finally, the characterization of the various cell and face gradient options is performed on the zero pressure gradient flat plate case. 


\section{Overview of USM3D}

The current USM3D [7, 8] code is a parallelized tetrahedral cell-centered, finite volume Navier-Stokes flow solver. The term "cell centered" means that the flow variables are solved at the centroid of each tetrahedral cell. Inviscid flux quantities are computed across each tetrahedral cell face using various upwind schemes. Spatial discretization is accomplished by a novel reconstruction process, based on an analytical formulation for computing solution gradients within tetrahedral cells. The solution is advanced in time by a $2^{\text {nd }}$-order Newton time step scheme [9], or to a steady-state condition by an implicit backward-Euler scheme. Several turbulence models are available: the Spalart-Allmaras (SA) one-equation model, the two-equation $k-\varepsilon$ turbulence model, the Menter Shear Stress Transport (SST) two-equation model, and the nonlinear Algebraic Reynolds Stress Models (ARSM) of Girimaji and Shih/Zhu/Lumley $[8,10,11]$. Detached Eddy Simulation (DES) has been implemented in all of the turbulence models. A capability to trip the flow at specified locations on aerodynamic surfaces has been implemented for the $k$ -

$\varepsilon$ turbulence model, but fully turbulent flow is assumed for the results to follow. USM3D has capabilities for dynamic grid motion and overset grids [12].

\section{Mixed Element Extensions to USM3D}

For second-order spatial accuracy in a cell-centered finite volume Navier-Stokes flow solver, gradients of solution variables are needed within a cell as well as on the faces of a cell. The gradients are used for the computation of convective and diffusive fluxes for the mean flow and turbulence model equations, as well as for the turbulence model source terms. Several changes have been incorporated in the tetrahedral grid version of USM3D to extend it for mixed element grids. Solution reconstruction based on an analytical formulation [19] that is applicable only for tetrahedral cells has been substituted by an explicit Taylor series expansion of primitive variables within each cell. Consequently, the cell gradient of a solution variable is explicitly evaluated and stored in solver memory. The extended flow solver allows flexibility to compute cell and face gradients using any of the several methods described below. In this section, key elements of solution methodology will be summarized.

\section{A. Geometric Quantities}

Cell center coordinates are calculated from arithmetic averaging of the coordinates of nodes that define a cell. Similarly, face center coordinates are calculated from arithmetic averaging of the coordinates of face nodes. Directed area of a face is calculated from the directed areas of its sub-triangles formed using the face edges and the face center. Cell volume is calculated by summing up the volumes of its constituent tetrahedral cells. A constituent tetrahedron within a cell is constructed from a sub-triangle of a cell face and the center of the cell.

\section{B. Cell Gradient}

Several options have been implemented for the computation of a solution variable gradient within a cell. The options include Green-Gauss integration, unweighted or weighted least squares procedure, and Mitchell stencil [8].

In the Green-Gauss integration, solution variables at the center of a cell face are calculated based on the averaging of the solution variables at the face nodes. Solution variables at a node are interpolated from the cells surrounding the node using a weighted averaging procedure [19]. The weights in the averaging procedures can optionally be limited between 0 and 2 to avoid the violation of the principle of positivity and maintain solution stability. Figure 2 shows a schematic representation of an unstructured grid. With reference to Fig. 2, the GreenGauss stencil for cell A consists of all the cells shown in the figure (cells A, 1-13). This stencil is called a fully augmented stencil.

For a least squares procedure, cell gradient of a solution variable can be evaluated using stencils of differing size. For example, a nearest-neighbor stencil can be used that includes only the face-neighbor cells (cells A, 1, 2, 3). A nearest-neighbor stencil for a boundary cell comprises of ghost cells associated with all of its boundary faces. It is a very compact and computationally efficient stencil. However, for tetrahedral grids, a solution based on this stencil quickly becomes unstable. On the other hand, a fully augmented stencil consisting of all the cells surrounding each node of a cell can also be used in the least squares procedure. This stencil does not include ghost cells for a boundary cell. The stencil is stable for any cell topology, however it is computationally inefficient. An optimized smart augmentation [20] stencil that is stable yet efficient is also implemented, which is composed of cells A, 1, 2, 3, 5,8 , and 12, in our example. An option to use fully augmented stencil for the boundary cells, and either nearest neighbor, fully augmented, or smartly augmented stencil for the interior cells is available as well. For the weighted least squares, contributions to a solution variable gradient from any cell within the stencil of a specific cell is weighted by the inverse of the distance between the two cell centers under consideration. 


\section{Face Gradient}

Face gradient of a solution variable can be evaluated from either Mitchell's stencil [8] or an average of the gradients within two cells sharing the face. For the latter case, the cell-averaged gradient is suitably augmented using a face-normal augmentation [21], an edge-normal method [22], or a face-tangent [23] method. Various methods are illustrated next using a sketch below for a $2 \mathrm{D}$ grid.

The sketch shows a grid face defined by two nodes $\mathrm{p} 1$ and $\mathrm{p} 2$. The face is shared by cells C and D. A position vector $\vec{r}$ points from the center of cell $\mathrm{C}$ to the center of cell $\mathrm{D}$. The unit normal to the face is represented by $\hat{n}$. The face gradient computation procedure based on the Mitchell's stencil is shown in Eq. (1) below. The Eqs. (2-4) illustrate face gradient based on the face-normal, edge-normal, and face-tangent methods, respectively.

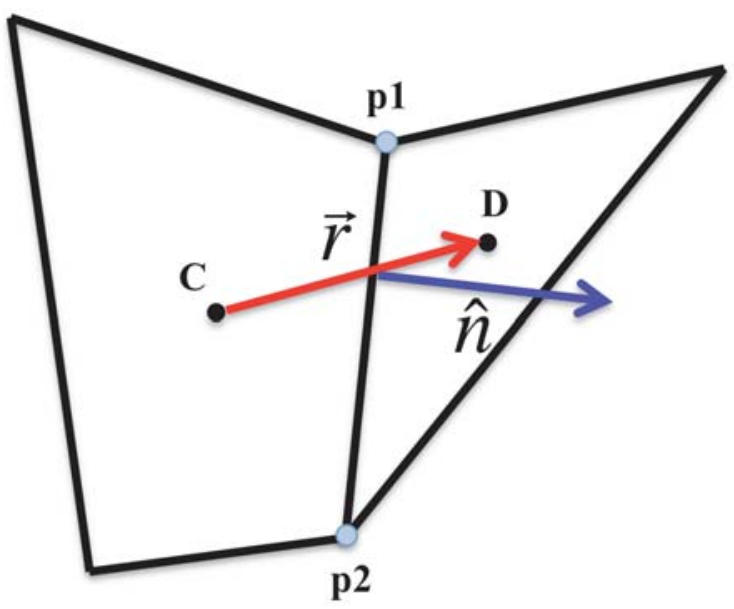

$$
\begin{aligned}
& {\left[\begin{array}{cc}
X_{D}-X_{C} & Y_{D}-Y_{C} \\
X_{p 2}-X_{p 1} & Y_{p 2}-Y_{p 1}
\end{array}\right]\left\{\begin{array}{l}
\phi_{X} \\
\phi_{Y}
\end{array}\right\}=\left\{\begin{array}{c}
\phi_{D}-\phi_{C} \\
\phi_{p 2}-\phi_{p 1}
\end{array}\right\}} \\
& \nabla \phi_{f}=\nabla \phi_{\text {avg }}+\left[-\left(\nabla \phi_{\text {avg }} \cdot \hat{n}\right)+\frac{\phi_{D}-\phi_{C}}{\vec{r} \cdot \hat{n}}\right] \hat{n} \\
& \nabla \phi_{f}=\nabla \phi_{\text {avg }}+\left[-\left(\nabla \phi_{\text {avg }} \cdot \hat{r}\right)+\frac{\phi_{D}-\phi_{C}}{|\vec{r}|}\right] \hat{r} \\
& \nabla \phi_{f}=\nabla \phi_{\text {avg }}+\frac{1}{\hat{n} \cdot \hat{r}}\left[-\left(\nabla \phi_{\text {avg }} \cdot \hat{r}\right)+\frac{\phi_{D}-\phi_{C}}{|\vec{r}|}\right] \hat{n}
\end{aligned}
$$

$\hat{r}$ is the unit vector along the position vector $\vec{r}$, such that $\hat{r}=\vec{r} /|\vec{r}|$. In the Eqs. (1-4), $\nabla \phi_{f}=\phi_{X} \vec{i}+\phi_{Y} \vec{j}$ is the gradient of a scalar function $\phi . \nabla \phi_{\text {avg }}$ represents the average of the gradients in cell $\mathrm{C}$ and $\mathrm{D}$; $\nabla \phi_{\text {avg }}=\left(\nabla \phi_{C}+\nabla \phi_{D}\right) / 2$

\section{Turbulence Models}

The Spalart-Allmaras [24] and the Menter Shear Stress Transport [25] turbulence models have been implemented to support mixed element grids. Various researchers have attempted several revisions to these two models. The standard forms of these models as well as some of the noteworthy revisions to them have been comprehensively documented in the NASA Langley Research Center Turbulence Model Resource website [26]. Following the nomenclature used in the website, "standard" Spalart-Allmaras One-Equation Model (SA), "Standard" Menter SST Two-Equation Model (SST), Menter SST Two-Equation Model with Vorticity Source Term (SST-V), and Menter 2003 SST Two-Equation Model (SST-2003) are presently available in the extended version of USM3D.

\section{Verification Results}

USM3D extensions for mixed element grids are verified using three verification cases available on the Turbulence Model Resource website [26]. All the cases are quasi two-dimensional (2D), having one hexahedral cell in the spanwise direction. The cases are zero pressure gradient flat plate, bump-in-channel, and planar shear. For each case, a series of five nested structured grids in PLOT3D and CGNS formats are available on the Turbulence Model Resource website [26]. A utility is used to convert structured PLOT3D grids into unstructured hexahedral grids and write them in the VGRID . gridu format. USM3D solutions are computed based on the unstructured hexahedral grids. Grid convergence of certain local as well as integrated quantities is monitored. Additional USM3D verification is achieved by code-to-code comparison. For this purpose, the finest grid USM3D and CFL3D [27] solutions are compared. The CFL3D results shown in this paper are obtained from the Turbulence Model Resource website. Verification results are presented in the sub-sections A, B, and C below.

Grid topology effects on a solution are studied using a 2D planar shear case in sub-section D. For this purpose, grid convergence of various quantities from the hexahedral, prismatic, and tetrahedral grid solutions is presented.

4

American Institute of Aeronautics and Astronautics 
Each of these grid types is generated from the same underlying structured grids. In sub-section E, three gradient computation options are assessed using the 2D zero pressure gradient flat plate case.

USM3D computations reported in the present work treat turbulence model convective terms with first-order spatial accuracy. Inviscid flux quantities are computed using Roe's flux-difference-splitting (FDS) scheme. Steadystate solutions are obtained using local time stepping and implicit defect-correction scheme. USM3D solutions for all three verification cases have been computed using the "standard" Spalart-Allmaras One-Equation Model (SA) as well as the Menter SST Two-Equation Model with Vorticity Source Term (SST-V). However, for the sake of brevity, only the results from the SA model will be presented in the present paper.

USM3D results in sub-sections A-D are based on the "GGM" option that uses Green-Gauss integration procedure for the computation of gradients within a cell and the Mitchell's method for the computation of gradients at a face. In the GGM method, cell gradients are used for the evaluation of mean flow convective terms whereas the face gradients are used for the mean flow and turbulence model diffusive terms as well as the turbulence model source terms.

\section{A. 2D Zero Pressure Gradient Flat Plate}

For this case, five progressively finer unstructured hexahedral grids have been generated that are based on the underlying structured PLOT3D grids. The specific nested structured grids [26], denoted by the number of grid points in the spanwise, streamwise, and body-normal directions, are $2 \times 35 \times 25,2 \times 69 \times 49,2 \times 137 \times 97,2 \times 273 \times 193$, and $2 \times 545 \times 385$. Solutions have been computed for the freestream Mach number of 0.2 and Reynolds number of $5 \times 10^{6}$ per unit length of the plate. The angle-of-attack is zero degree. Figure 3 presents a schematic view of the computational domain and the boundary conditions in the X-Z plane. Symmetry boundary condition is applied on the two spanwise planes.

On each grid, a USM3D solution is computed starting from the freestream-based initial conditions. The solutions are computed using a progressively larger number of iterations to ensure accurate results on all grids. The number of iterations ranged from 25,000 for the coarsest grid to 150,000 for the finest grid. The convergence of the USM3D mean flow and SA model residual errors is presented in Fig. 4 for all five grids. It is evident from the figure that on all grids the rms of the mean flow residual errors is below 1.0e-15 and the SA model residual errors is below 1.0e12.

USM3D verification results based on the SA model are presented next. Figure 5 shows grid convergence of the USM3D and CFL3D computed skin friction at a specific location on the plate as well as the drag coefficient integrated over the plate. It can be seen that as the grid is refined, USM3D and CFL3D results show closer agreement. Local skin friction and the integrated drag from USM3D and CFL3D are almost identical on the finest grid. It should be noted that the coarsest grid USM3D and CFL3D results appear very different due to a narrow yaxis range. For example, on the coarsest grid USM3D and CFL3D drag values differ only by less than 1.2 count.

In the next two figures the finest grid USM3D and CFL3D results are compared. The local skin friction variations along the plate are compared in Fig. 6, whereas, the profiles of streamwise velocity and eddy viscosity in the body-normal direction are presented in Fig. 7. The streamwise velocity profiles are presented in terms of the inner wall variables $\left(\mathrm{u}^{+}\right.$and $\left.\mathrm{y}^{+}\right)$. The profiles are presented at two streamwise stations on the plate (Fig. 7a). The eddy viscosity variations across the boundary layer are compared at one streamwise station on the plate (Fig. 7b). Figures 6 and 7 demonstrate almost identical results from USM3D and CFL3D.

\section{B. 2D Bump-in-Channel}

For this case, five progressively finer unstructured hexahedral grids have been generated that are based on the underlying structured PLOT3D grids. The specific nested structured grids [26], denoted by the number of grid points in the spanwise, streamwise, and body-normal directions, are $2 \times 89 \times 41,2 \times 177 \times 81,2 \times 353 \times 161,2 \times 705 \times 321$, and $2 \times 1409 \times 641$. Solutions have been computed for the freestream Mach number of 0.2 , Reynolds number of $3 \times 10^{6}$ per unit grid length, and angle-of-attack of 0 degree. Figure 8 presents a schematic view of the computational domain and the boundary conditions in the X-Z plane. Symmetry boundary condition is applied on the two spanwise planes.

On each grid, USM3D solution is computed starting from the freestream-based initial conditions. Moving from the coarsest to the finest grid, the solutions required a progressively larger number of iterations to converge. USM3D is run for 40,000 iterations on the coarsest grid and for as many as 500,000 iterations on the finest grid. The convergence of the USM3D mean flow and SA model residual errors is presented in Fig. 9 for all five grids. The rms of the residual errors is reduced below 1.0e-12 for the mean flow equations and below 1.0e- 6 for the SA model equation. On the finest two grids, the USM3D mean flow and SA model convergence is very slow, possibly due to the linear solver that is first-order accurate and uses point-implicit algorithm. This issue will be investigated in the follow-on work by using a consistent linearization and line-implicit scheme for the linear solver. 
USM3D SA model verification results for this case are presented in Figs. 10-14. Grid convergence of the USM3D and CFL3D computed local skin friction coefficient at the bump apex and the integrated viscous drag coefficient is displayed in Fig. 10. Figure 11 presents the grid convergence of the total drag and lift coefficients from the USM3D and CFL3D solutions. Except for the viscous drag coefficient, all the quantities in Figs. 10 and 11 exhibit similar convergence trends. The USM3D and CFL3D results on the coarsest grid differ the most. For example, the total drag from USM3D and CFL3D differs by about seven counts on the coarsest grid. However, as in the flat plate case, the skin friction and the integrated force coefficients converge to nearly identical values on the finest three levels of grid.

Next, the finest grid USM3D and CFL3D solutions are compared. Figure 12 presents the streamwise variations of the skin friction and the pressure coefficient along the bump surface. In Fig. 13, streamwise velocity and eddy viscosity variations in the direction normal to the bump surface are shown at certain stations on the bump. The eddy viscosity field variations from the USM3D and CFL3D solutions are demonstrated in Fig. 14. For the sake of ready comparison, the contour plots in Fig. 14 use the identical range for both the solutions. The finest grid USM3D and CFL3D results as presented in Figs. 12-14 demonstrate an excellent agreement between the two flow solvers.

\section{2D Planar Shear}

This case involves the growth of a free shear layer that develops when two different streams passing over a thin plate begin mixing. For this case, five progressively finer single-block unstructured hexahedral grids have been generated that are based on the underlying multi-block structured PLOT3D grids. Each structured grid consists of three blocks. In the description below, dimensions of all blocks will be listed parenthetically to represent a blockstructured grid. The specific nested structured grids [26], denoted by the number of grid points in the spanwise,

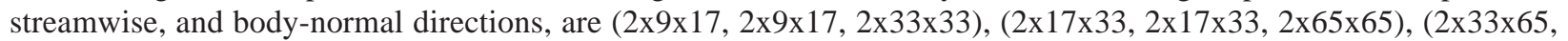
$2 \times 33 \times 65,2 \times 129 \times 129),(2 \times 65 \times 129,2 \times 65 \times 129,2 \times 257 \times 257)$, and $(2 \times 129 \times 257,2 \times 129 \times 257,2 \times 513 \times 513)$. Figure 15 presents a schematic view of the computational domain and the boundary conditions in the X-Z plane. Symmetry boundary condition is imposed on the two spanwise planes. The Mach number of the smaller stream below the plate is 0.5 , whereas, the Mach number of the larger stream above the plate is 0.25 . Solutions have been computed for the Reynolds number of 50,000 based on unit grid length.

For each grid, USM3D solution is computed starting from the freestream-based initial conditions. USM3D is run for 20,000 iterations each on the coarsest two levels of grids. For the next two finer levels of grid USM3D is run for 30,000 and 50,000 iterations, respectively. On the finest grid, USM3D is run for 125,000 iterations. The convergence histories of the mean flow and SA model equation residual errors are similar to those for the flat plate case and therefore not shown here. It is noted that the rms of the USM3D residual errors on all grids is reduced below 1.0e-14 for the mean flow equations and below 1.0e-10 for the SA model equation.

USM3D SA model verification results for this case are presented in Figs. 16-20. Grid convergence of the USM3D and CFL3D solutions is exhibited in Fig. 16 using two quantities, namely, the drag coefficient on the streams-dividing plate and the streamwise velocity at a specific location in the computational domain. The finest grid drag coefficient from USM3D and CFL3D differs by a little over $1 / 2$ count. The local streamwise velocity from USM3D and CFL3D are seen to be converging to similar values.

Similar to the previous two test cases, USM3D solution on the finest grid is assessed next using the corresponding CFL3D solution as the benchmark. Accordingly, the streamwise variations of streamwise velocity along the $\mathrm{Z}=0$ line are compared in Fig. 17. The normal-direction variations of streamwise velocity and eddy viscosity at a specific streamwise station are presented in Fig. 18. Next, a qualitative comparison of the USM3D and CFL3D computed flowfields is made. For this purpose, eddy viscosity variations in a spanwise plane are shown in Figs. 19-20. The variations in the proximity of the streams-dividing plate are presented in Fig. 19, whereas the farfield variations are demonstrated in Fig. 20. Based on the Figs. 17-20, it is observed that the USM3D and CFL3D solutions on the finest grid are in excellent agreement.

\section{Effect of Grid Topology}

The grid topology effect on the computed solutions is assessed using the 2D planar shear case. Based on the family of five unstructured hexahedral grids used previously, three additional sets of grids are generated by subdividing hexahedral cells in various manners. Each of these sets consists of only one type of cells. Two sets consist of prismatic cells whereas the third one consists of tetrahedral cells. Two prismatic cell sets differ by the direction in which prism cells are aligned. In one set, prismatic cells are aligned in the direction normal to the streams-dividing plate. This set will be identified as "Normal prisms" in the discussion to follow. Another set, designated as "Sideways prisms", comprises of prismatic cells that are aligned in the spanwise direction. The original hexahedral grid set will be called "Hexes", whereas the one made up of tetrahedral cells will be named as 
"Tets". Using the finest grid in the respective sets, the characteristics of various sets are summarized in the Table 1 below.

Table 1. Statistics for the finest grid in various grid sets constructed for the planar shear case

\begin{tabular}{|c|c|c|c|c|}
\hline Grid Set Identifier & Number of Nodes & Number of Cells & $\begin{array}{c}\text { Number of } \\
\text { Boundary Faces }\end{array}$ & Number of Faces \\
\hline Hexes & 657,922 & 327,680 & 657,920 & $1,312,000$ \\
\hline Normal Prisms & 657,922 & 655,360 & 659,456 & $1,968,128$ \\
\hline Sideways Prisms & 657,922 & 655,360 & $1,313,280$ & $2,295,040$ \\
\hline Tets & 657,922 & $1,966,080$ & $1,315,840$ & $4,590,080$ \\
\hline
\end{tabular}

As in the previous cases, USM3D solution for each grid is computed starting from the freestream-based initial conditions. Following the grid notation system in Ref. [26], the five grids within each grid topology are identified by an integer number varying from 0 to 4 , where 0 is used to denote the finest grid whereas 4 is used to denote the coarsest grid. Using this identification system the convergence characteristics of three grid levels within various grid topologies are outlined in Table 2.

Table 2. Convergence characteristics of various grid sets constructed for the planar shear case

\begin{tabular}{|c|c|c|c|c|}
\hline Grid Set Identifier & Grid Level & Number iterations & $\begin{array}{c}\text { rms of mean flow } \\
\text { residuals }\end{array}$ & $\begin{array}{c}\text { rms of turbulence } \\
\text { model residuals }\end{array}$ \\
\hline Hexes & 4 & 20,000 & $1.84 \mathrm{e}-15$ & $5.96 \mathrm{e}-14$ \\
& 2 & 30,000 & $5.01 \mathrm{e}-16$ & $1.99 \mathrm{e}-14$ \\
& 0 & 125,000 & $5.13 \mathrm{e}-16$ & $6.01 \mathrm{e}-10$ \\
\hline Normal Prisms & 4 & 20,000 & $4.20 \mathrm{e}-15$ & $7.87 \mathrm{e}-14$ \\
& 2 & 30,000 & $5.12 \mathrm{e}-16$ & $1.12 \mathrm{e}-14$ \\
& 0 & 170,000 & $1.27 \mathrm{e}-16$ & $1.24 \mathrm{e}-14$ \\
\hline Sideways Prisms & 4 & 20,000 & $2.03 \mathrm{e}-15$ & $3.21 \mathrm{e}-3$ \\
& 2 & 30,000 & $5.00 \mathrm{e}-14$ & $6.46 \mathrm{e}-4$ \\
& 0 & 240,000 & $2.67 \mathrm{e}-12$ & $6.87 \mathrm{e}-5$ \\
\hline Tets & 4 & 20,000 & $2.85 \mathrm{e}-15$ & $3.91 \mathrm{e}-4$ \\
& 2 & 60,000 & $1.62 \mathrm{e}-14$ & $1.31 \mathrm{e}-4$ \\
\end{tabular}

It is evident from Table 2 that the convergence behavior of "Normal Prisms" is similar to "Hexes" and "Tets" is similar to "Sideways Prisms". The SA model convergence for the "Sideways Prisms" and "Tets" is unsatisfactory across all grid levels. Examination of the evolution of the SA model primary variable for the latter two grid topologies reveals that the variable rapidly approaches its artificially imposed minimum value of $1.0 \mathrm{e}-10$ inside USM3D. The clipping of the SA model variable may be responsible for the stalled convergence. A separate study focusing on the implementation and evaluation of the "negative" SA model [28] is underway to examine a possible link between the artificial clipping of the SA model variable and a degraded convergence of the model.

Grid convergence of USM3D solutions on various grid topologies is monitored in Fig. 21. For this purpose, the variations of drag coefficient on the streams-dividing plate and streamwise velocity at a specific location in the computational domain are plotted. It can be seen from Fig. 21 that with grid refinement all grid topologies converge to similar values.

USM3D finest grid solutions from various grid topologies are examined next using the streamwise variations of streamwise velocity along the $\mathrm{Z}=0$ line (Fig. 22) and the normal-direction variations of streamwise velocity and eddy viscosity at a specific streamwise station in the computational domain (Fig. 23). It is clear from Figs. 22-23 that the finest grids solutions from various grid topologies are in close agreement.

\section{E. Assessment of Various Gradient Computation Options}

In this sub-section the $2 \mathrm{D}$ zero pressure gradient flat plate case is revisited to compare hexahedral grid solutions obtained by exercising various USM3D options for the computation of cell and face gradients. In addition to the USM3D "GGM" solutions reported in sub-section A, two additional USM3D solution sets have been computed for the same case. 
Both of the additional sets use least squares procedure for the computation of gradients within a cell. One set, designated as "ULSQ", uses unweighted least squares procedure whereas the "WLSQ" solution set uses the weighted least squares procedure. In both the sets, cell gradients are used for the evaluation of mean flow convective terms as well as the turbulence model source terms. In both sets, face gradients are computed from the average of respective cell gradients that are augmented by the "face-tangent" method. The mean flow and turbulence model diffusive terms are computed using these face gradients.

On all five hexahedral grids, USM3D "ULSQ" and "WSLQ" solutions are computed using the same number of iterations as that used for the respective USM3D "GGM" solutions. The convergence of the mean flow and SA model in the solutions computed from the three options is very similar and therefore not shown here. For example, in the finest hexahedral grid USM3D "ULSQ", "WLSQ", and "GGM" solutions, the rms of the mean flow residuals ranged from $1.53 \mathrm{e}-15$ to $1.54 \mathrm{e}-15$ and the rms of the SA model residuals varied from $2.61 \mathrm{e}-13$ to $2.77 \mathrm{e}-13$.

Figure 24 shows the grid convergence of skin friction at a specific location on the plate and the drag coefficient integrated over the plate, both of which are computed using various USM3D options described earlier. Local skin friction and integrated drag show close agreement among various USM3D solutions on the finest two grids. It can be seen from Fig. 24 that the local skin friction and the integrated drag from USM3D "WLSQ" and "GGM" solutions are in close agreement on all grids. However, the local skin friction and the integrated drag in the USM3D "ULSQ" solution are significantly lower on the coarsest grid. For example, on the coarsest grid, "ULSQ" drag is approximately three counts lower than the corresponding "WLSQ" and "GGM" values.

In the next two figures the finest grid results from the three USM3D solutions are compared. Variations of local skin friction along the plate are plotted in Fig. 25, whereas, the profiles of streamwise velocity and eddy viscosity in the body-normal direction are presented in Fig. 26. The streamwise velocity profiles (Fig. 26a) at two streamwise stations are shown in terms of the inner wall variables $\left(\mathrm{u}^{+}\right.$and $\left.\mathrm{y}^{+}\right)$. The eddy viscosity variations (Fig. 26b) are compared across the boundary layer at one streamwise station on the plate. The results in Figs. 25 and 26 display almost identical agreement between the three USM3D solutions.

\section{Concluding Remarks}

The NASA USM3D tetrahedral grid cell-centered finite volume flow solver has been recently extended to accommodate mixed element grids composed of hexahedral, prismatic, pyramidal, and tetrahedral cells. The important work of code verification is well underway. The initial verification study that is the topic of this paper used three two-dimensional test cases available on the Turbulence Modeling Resource website at the NASA Langley Research Center; the zero pressure gradient flat plate, planar shear, and bump-in-channel. The approach was to first verify the implementation on hexahedral grids through direct comparison to solutions from the well-established structured-grid code CFL3D. Next the effect of cell topologies on the flow solution was assessed using the planar shear case. And finally, the various cell and face gradient reconstruction schemes were evaluated on the zero pressure gradient flat plate case using hexahedral grids.

The integrity of coding implementations is verified through assessments of convergence for the flow and turbulence model equations, grid-convergence of both integrated and distributed pressure and skin-friction coefficients, and off-body comparisons of velocity and eddy viscosity distributions. The overall integrity of USM3D code modifications, which include a more general data structure, implementation of least-squares reconstruction, and the generalization of Green-Gauss scheme, is confirmed by near identical agreement with CFL3D solutions on hexahedral grids for all criteria. The only anomaly to surface was a stalled convergence on tetrahedral and "sideways" prisms for planar shear flow that may be caused by an artificial clipping inside the USM3D implementation of Spalart-Allmaras (SA) turbulence model. A separate study of a "negative" SA model is underway to investigate this problem.

While the current verification study only included the SA model results, equally good correlations have been obtained with the Shear-Stress Transport (SST) model, but were not included for sake of brevity. These results will be subject of a future publication.

\section{Acknowledgments}

The work reported herein is funded by the Vehicle Systems Safety Technologies (VSST) project under the NASA Aviation Safety Program, and the Subsonic Fixed Wing (SFW) project under the NASA Fundamental Aeronautics Program. The authors would like to thank Dr. Christopher Rumsey of NASA Langley Research Center for providing a utility to convert structured multiblock PLOT3D grid in to an unstructured grid format and for many helpful discussions related to turbulence models. Authors would also like to thank Dr. Jim Thomas of NASA 
Langley Research Center and Dr. Boris Diskin of National Institute of Aerospace for several fruitful discussions to improve the accuracy of numerical gradients. Dr. Khaled Abdol-Hamid of NASA Langley Research Center generated laminar flat plate solutions using PAB3D that helped verify initial USM3D solutions.

\section{References}

[1] Frink, N. T., Pirzadeh, S. Z., Parikh, P. C., Pandya, M. J., and Bhat, M. K., "The NASA tetrahedral unstructured software system (TetrUSS)," The Aeronautical Journal, Vol. 104, No. 1040, 2000, pp. 491-499.

[2] Pandya, M. J., Frink, N. T., Abdol-Hamid, K. S., Samareh, J. A., Parlette, E. B., and Taft, J. R., "Enhancements to TetrUSS for NASA Constellation Program," Journal of Spacecraft and Rockets, Vol. 49, No. 4, 2012, pp. 617-631.

[3] Samareh, J. A., "GridTool: A Surface Modeling and Grid Generation Tool," Proceedings of the Workshop on Surface Modeling, Grid Generation, and Related Issues in CFD Solutions, NASA CP-3291, 9-11 May, 1995.

[4] Lohner, R. and Parikh, P., "Three-dimensional grid generation by the advancing front method," International Journal for Numerical Methods in Fluids," Vol. 8, Issue 10, 1988, pp. 1135-1149.

[5] Pirzadeh, S. Z., "Three-dimensional unstructured viscous grids by the advancing layer method," AIAA Journal, Vol. 33, No. 1, 1996, pp. 43-49.

[6] Pirzadeh, S. Z., "Advanced unstructured grid generation for complex aerodynamic applications," AIAA Journal, Vol. 48, No. 5, 2010, pp. 904-915.

[7] Frink, N. T., "Upwind scheme for solving the Euler equations on unstructured tetrahedral meshes," $A I A A$ Journal, Vol. 30, No. 1, 1992, pp. 70-77.

[8] Frink, N. T., "Tetrahedral unstructured Navier-Stokes method for turbulent flows," AIAA Journal, Vol. 36 , No. 11, 1998, pp. 1975-1982.

[9] Pandya, M.J., Frink, N.T., Abdol-Hamid, K.S., and Chung, J.J., "Recent enhancements to USM3D unstructured flow solver for unsteady flows," AIAA Paper 2004-5201.

[10] Abdol-Hamid, K. S., Frink, N. T., Deere, K. A., and Pandya, M. J., "Propulsion Simulations Using Advanced Turbulence Models with the Unstructured-Grid CFD Tool, TetrUSS," AIAA Paper 2004-0714.

[11] Pandya, M. J., Abdol-Hamid, K. S., and Frink, N. T., "Enhancement of USM3D unstructured flow solver for high-speed high-temperature shear flows," AIAA Paper 2009-1329.

[12] Pandya, M.J., Frink, N.T., and Noack, R.W., "Overset-grid moving body capability in the USM3D unstructured flow solver," AIAA Paper 2005-5118.

[13] Campbell, R. L., "Efficient Viscous Design of Realistic Aircraft Configuration (Invited)," AIAA Paper 19982539.

[14] Bauer, S. X., Krist, S. E., and Compton, W. B. "Generation of the Ares I-X Flight Test Vehicle Aerodynamic Data Book and Comparison To Flight," AIAA Paper 2011-0011.

[15] Abdol-Hamid, K. S., Ghaffari, F., and Parlette, E. B., "Ares I Vehicle Computed Turbulent Ascent Aerodynamic Data Development and Analysis,” Journal of Spacecraft and Rockets, Vol. 49, No. 4, 2012, pp. 596-608.

[16] Green, B. E., "Computational Prediction of Nose-Down Control for F/A-18E at High Alpha," Journal of Aircraft, Vol. 45, No. 5, 2008, pp. 1661-1668.

[17] Hooker, J. R., “Aerodynamic Development of a Refueling Pod for Tanker Aircraft,” AIAA Paper 2002-2805.

[18] Thompson, J. R., Frink, N. T., and Murphy, P. C., "Guidelines for Computing Longitudinal Dynamic Stability Characteristics of a Subsonic Transport," AIAA Paper 2010-4819.

[19] Frink, N. T., "Recent Progress Toward a Three-Dimensional Unstructured Navier-Stokes Flow Solver," AIAA Paper 1994-0061.

[20] Diskin, B., and Thomas, J. L., "Comparison of Node-Centered and Cell-Centered Unstructured Finite Volume Discretization: Inviscid Fluxes,” AIAA Journal, Vol. 49, No. 4, 2011, pp. 836-854.

[21] Strang, W., Tomaro, R., and Grismer, M., "The Defining Methods of Cobalt60: A Parallel, Implicit, Unstructured Euler/Navier-Stokes Flow Solver,” AIAA Paper 1999-0786.

[22] Haselbacher, A., and Blazek, J., "Accurate and Efficient Discretization of Navier-Stokes Equations on Mixed Grids,” AIAA Journal, Vol. 38, No. 11, 2000, pp. 2094-2102.

[23] Diskin, B., and Thomas, J. L., "Comparison of Node-Centered and Cell-Centered Unstructured Finite Volume Discretization: Viscous Fluxes,” AIAA Journal, Vol. 48, No. 7, 2010, pp. 1326-1338. 
[24] Spalart P., and Allmaras S. A., "One-Equation Turbulence Model for Aerodynamic Flows," AIAA Paper 19920439.

[25] Menter, F.R., "Improved Two-Equation k-omega Turbulence Models for Aerodynamic Flows," NASA TM103975, October 1992.

[26] URL http://turbmodels.larc.nasa.gov.

[27] Krist, S. L., Biedron, R. T., and Rumsey, C. L., "CFL3D User's Manual (Version 5)," First Edition, Novermber 1996.

[28] Allmaras, S. R., Johnson, F. T., and Spalart, S. A., "Modifications and Clarifications for the Implementation of the Spalart-Allmaras Turbulence Model", ICCFD7-1902, Seventh International Conference on Computational Fluid Dynamics, Big Island, Hawaii, July 9-13, 2012. 


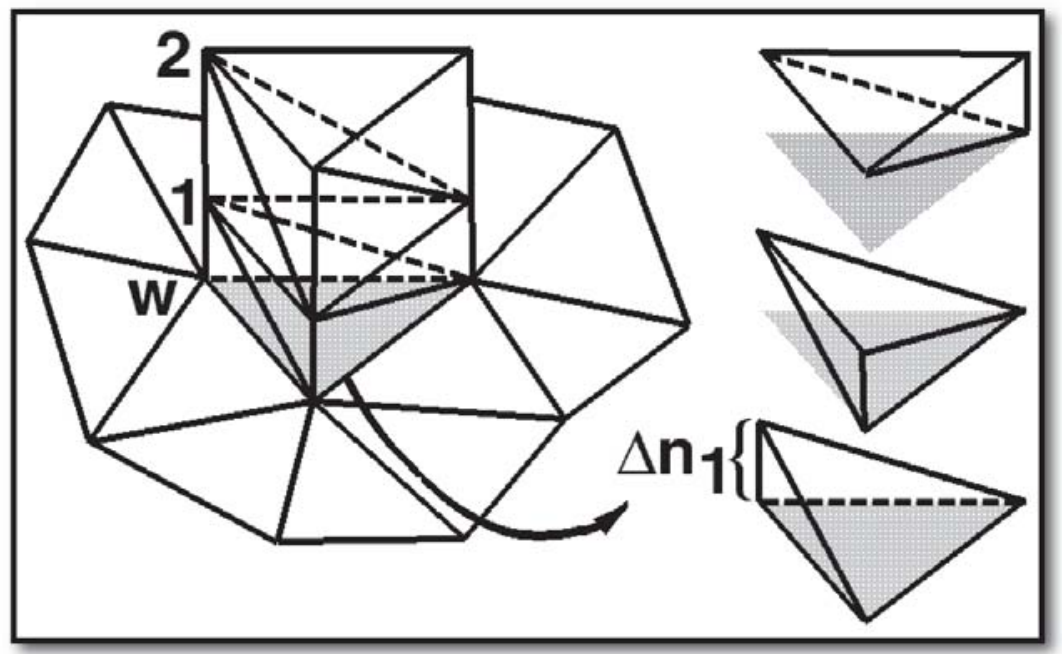

Figure 1. Thin-layered tetrahedral grid in the boundary layer region.

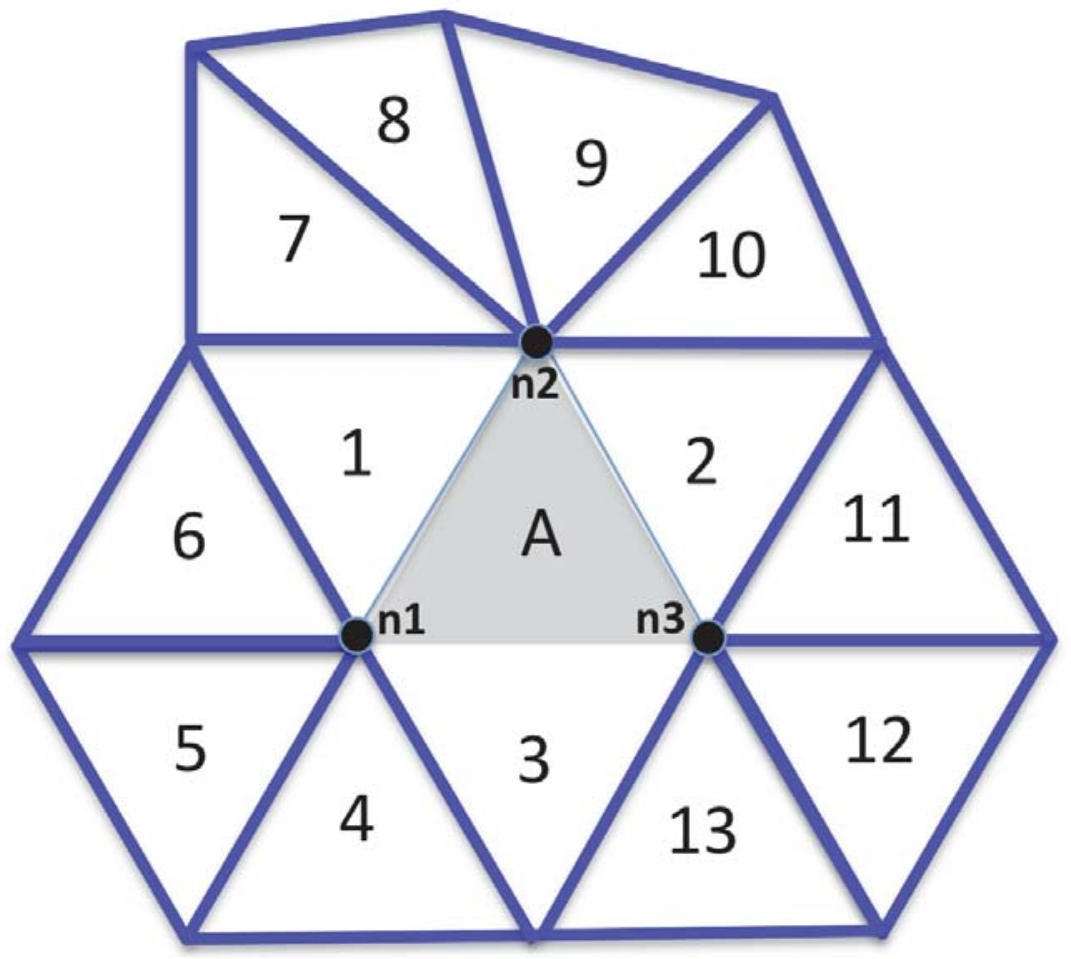

Figure 2. A representative view of a 2D unstructured triangular grid. 


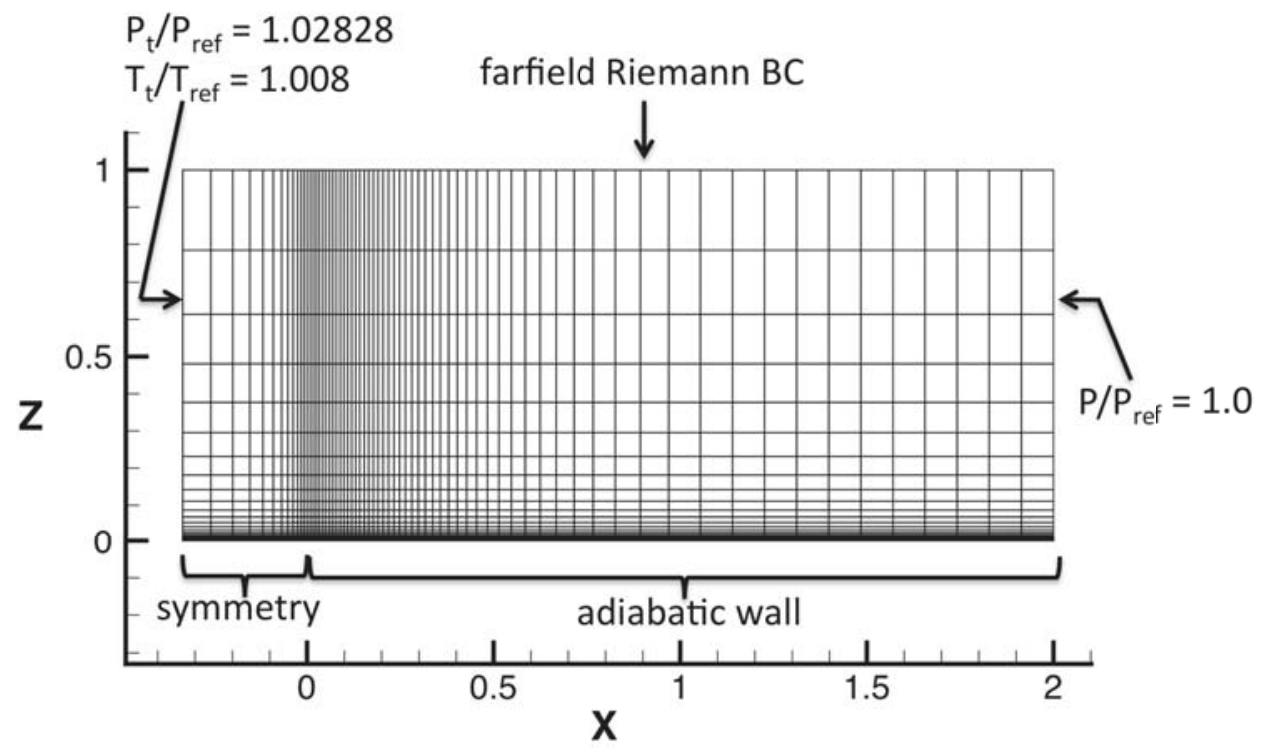

Figure 3. Schematic view of computational domain and various boundary conditions for the 2D zero pressure gradient flat plate.

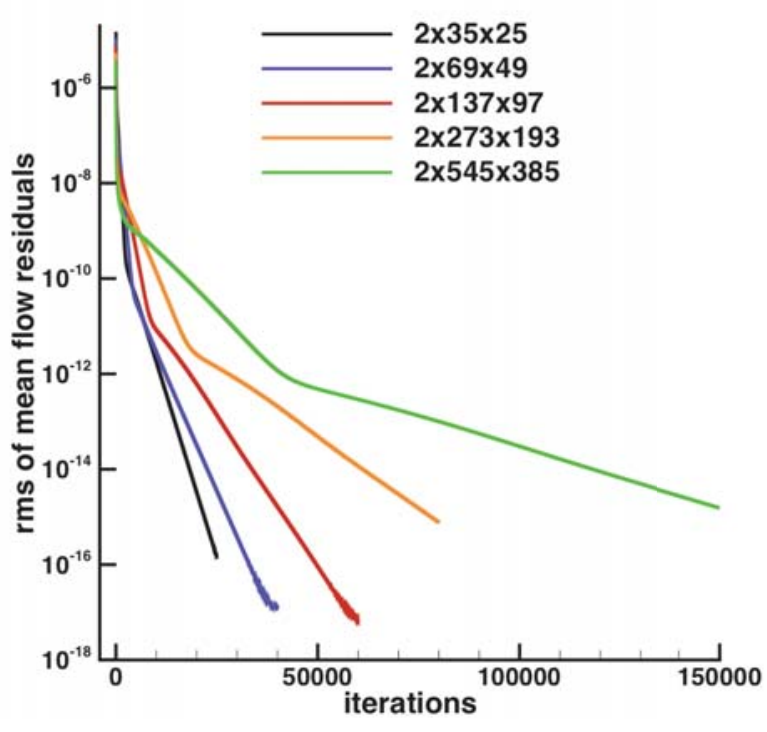

(a) residuals of mean flow equations

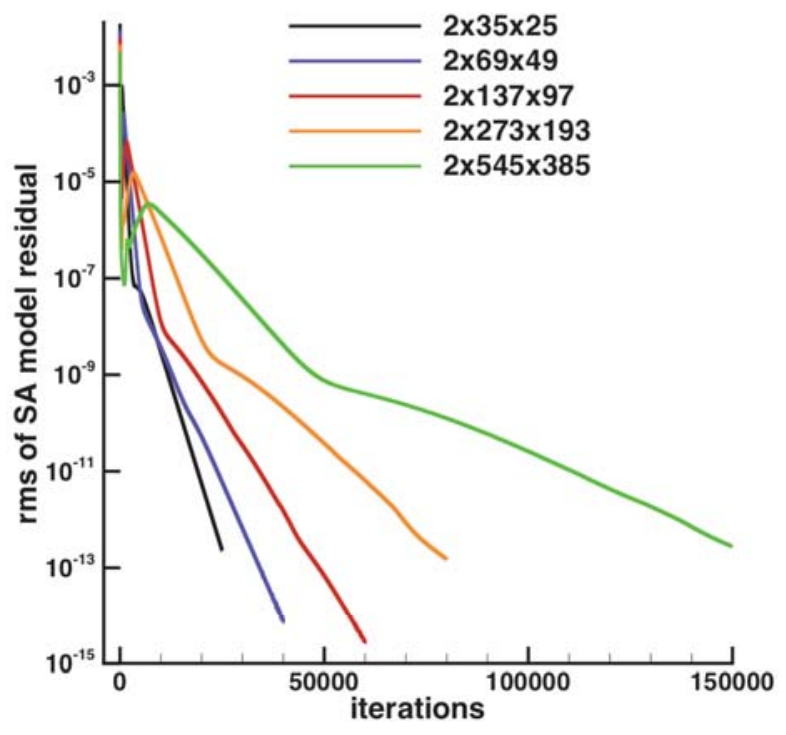

(b) residuals of SA model equation

Figure 4. USM3D convergence for the 2D zero pressure gradient flat plate on a sequence of five hexahedral grids. $M_{\infty}=0.2, \operatorname{Re}_{L}=5 \times 10^{6}(L=1)$. 


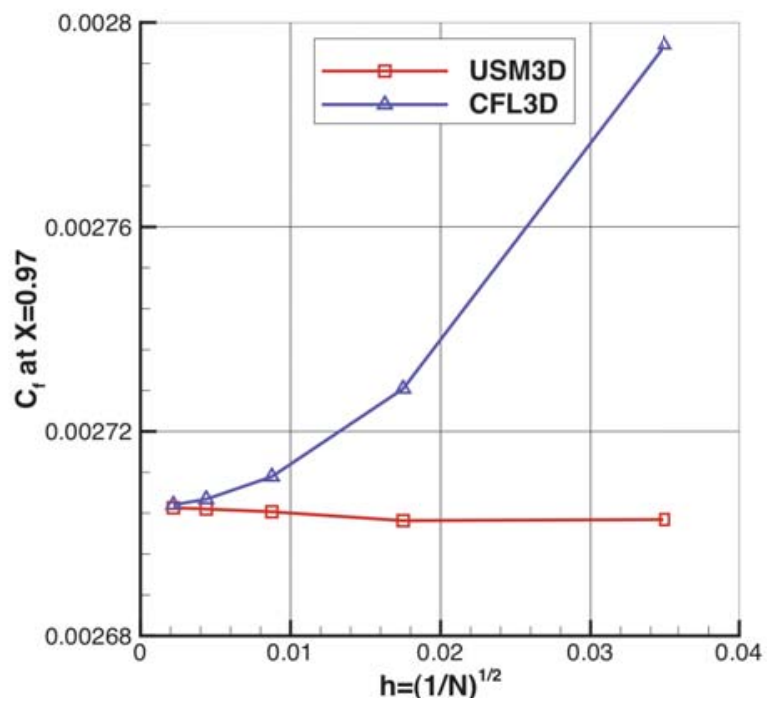

(a) local skin friction at $\mathrm{X}=0.97$

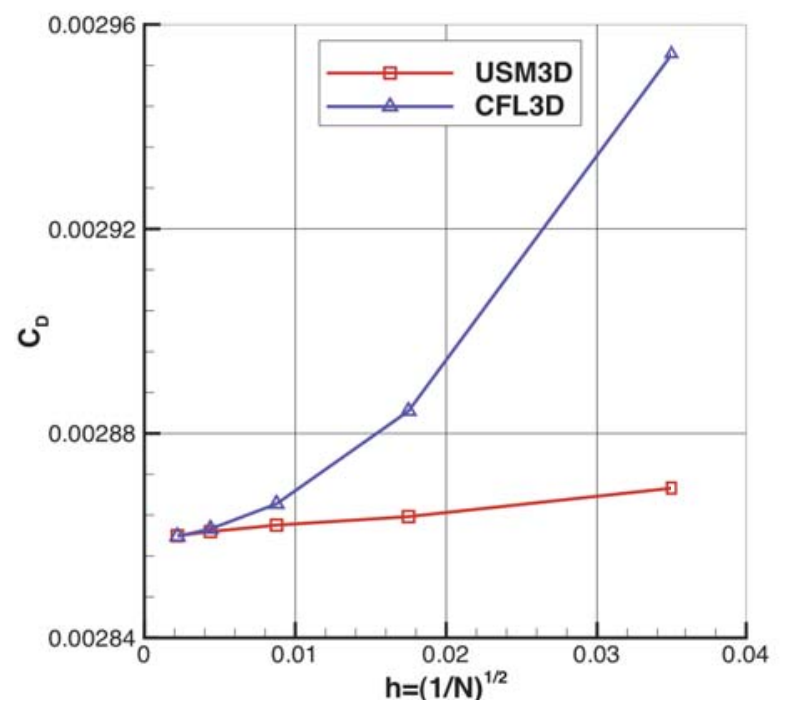

(b) integrated drag coefficient

Figure 5. Grid convergence of skin friction and drag coefficients for the 2D zero pressure gradient flat plate computed using SA model on a sequence of five hexahedral grids. $M_{\infty}=0.2, \operatorname{Re}_{\mathrm{L}}=5 \times 10^{6}(\mathrm{~L}=1)$.

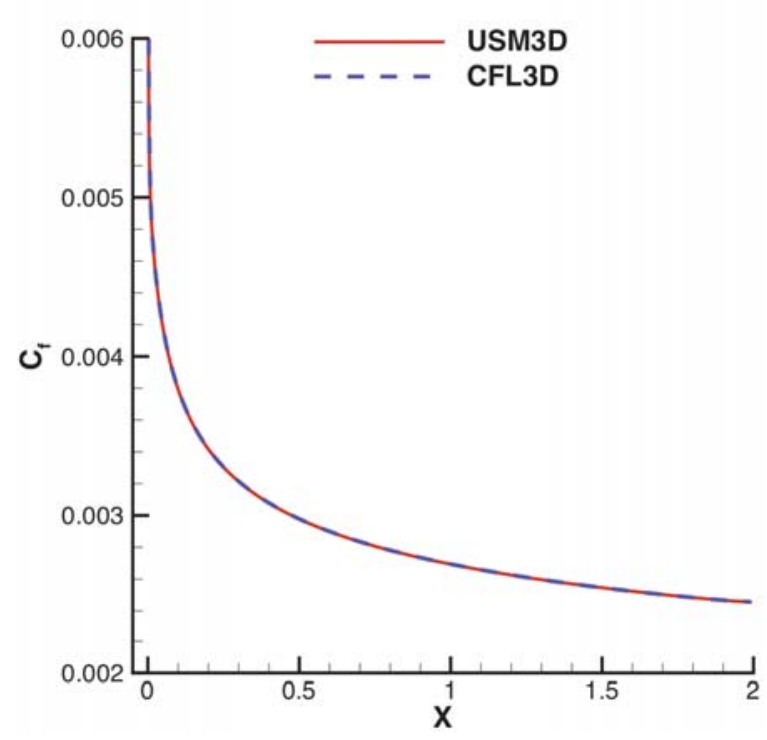

Figure 6. Comparison of the streamwise variations of local skin friction computed using SA model on the finest hexahedral grid for the $2 \mathrm{D}$ zero pressure gradient flat plate. $\mathrm{M}_{\infty}=0.2, \operatorname{Re}_{\mathrm{L}}=5 \times 10^{6}(\mathrm{~L}=1)$. 


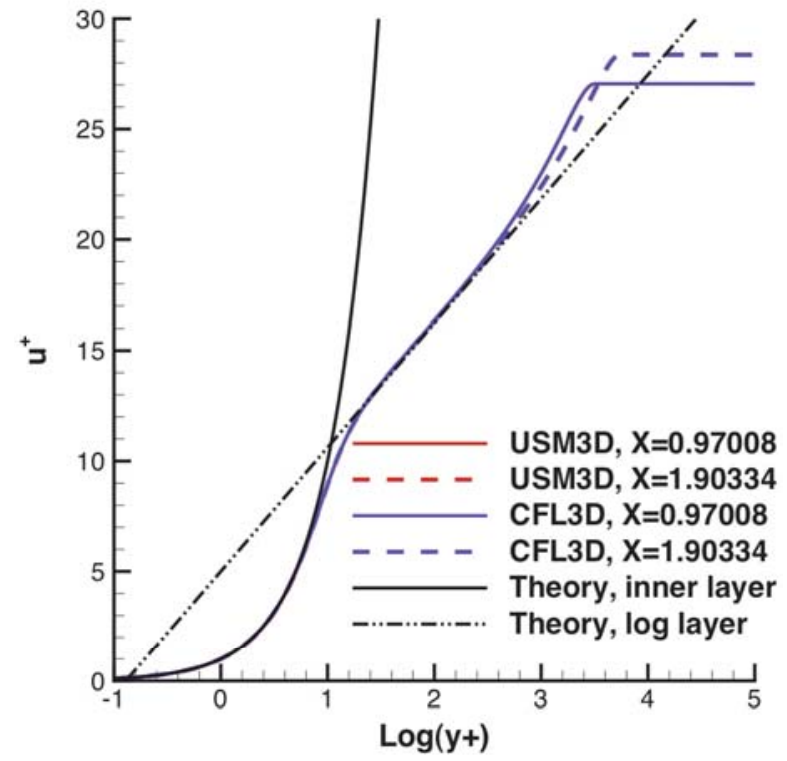

(a) streamwise velocity at two streamwise stations

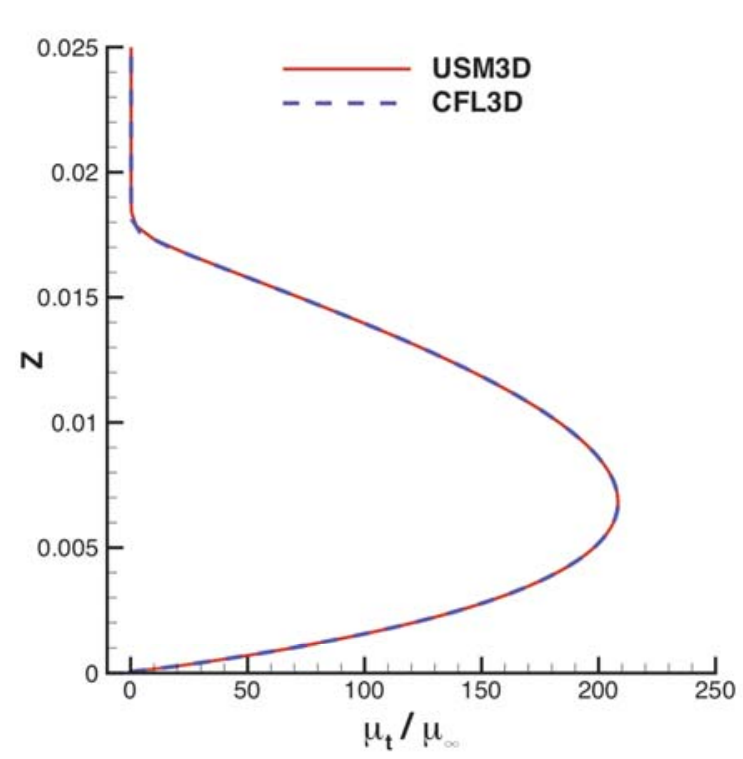

(b) eddy viscosity at $\mathrm{X}=0.97$

Figure 7. Comparison of the normal direction variations of streamwise velocity and eddy viscosity computed using SA model on the finest hexahedral grid for the $2 \mathrm{D}$ zero pressure gradient flat plate. $M_{\infty}=0.2, \operatorname{Re}_{\mathrm{L}}=5 \times 10^{6}$ $(\mathbf{L}=\mathbf{1})$.

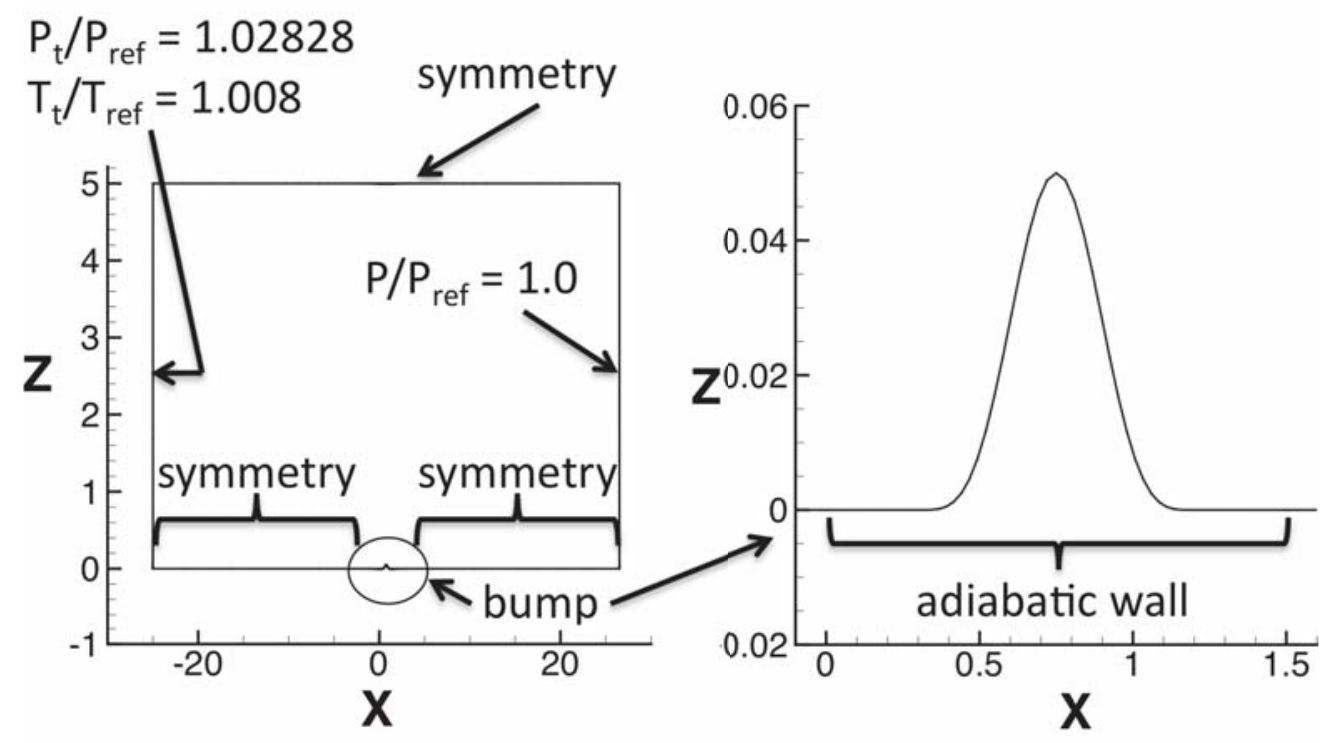

Figure 8. Schematic view of computational domain and various boundary conditions for the 2D bump-inchannel. 


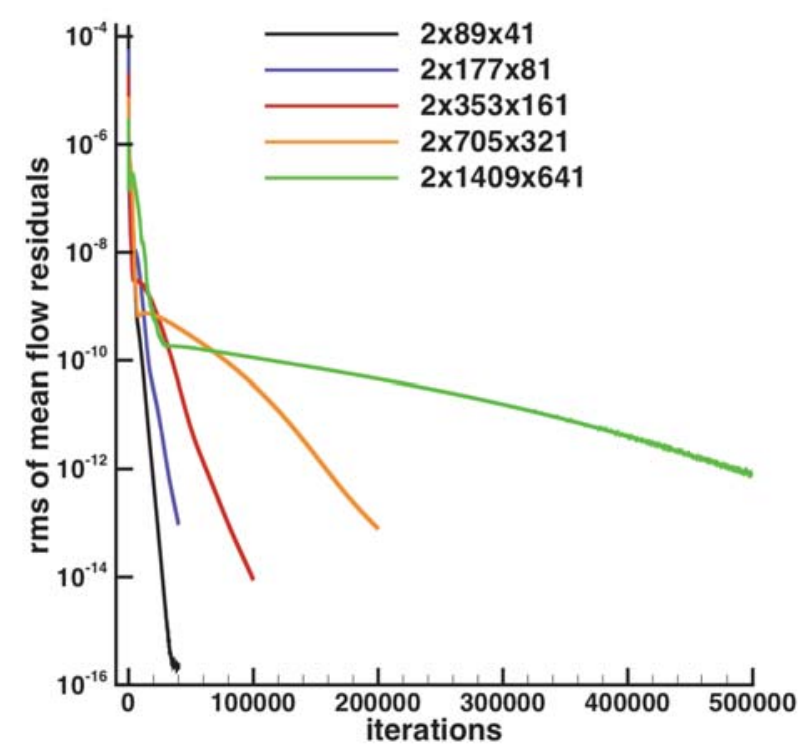

(a) residuals of mean flow equations

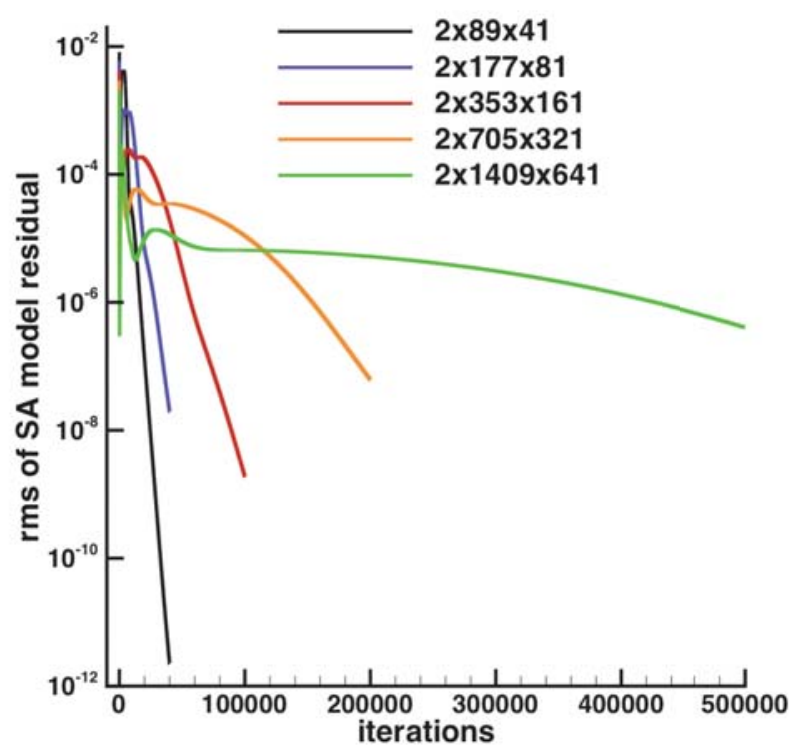

(b) residuals of $\mathrm{SA}$ model equation

Figure 9. USM3D convergence for the 2D bump-in-channel on a sequence of five hexahedral grids. $M_{\infty}=0.2$, $\operatorname{Re}_{\mathrm{L}}=3 \times 10^{6}(\mathrm{~L}=1)$.

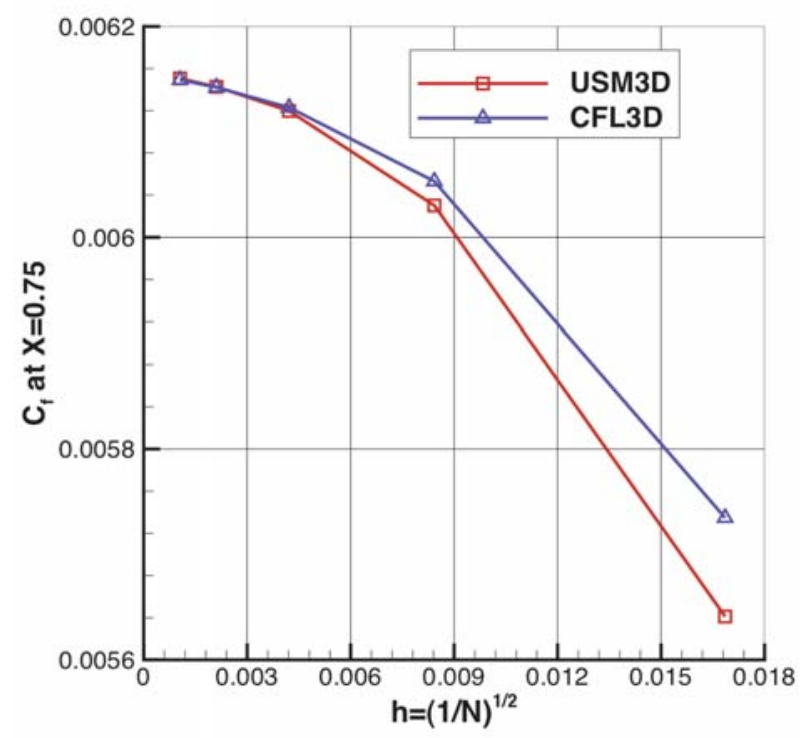

(a) local skin friction at $\mathrm{X}=\mathbf{0 . 7 5}$

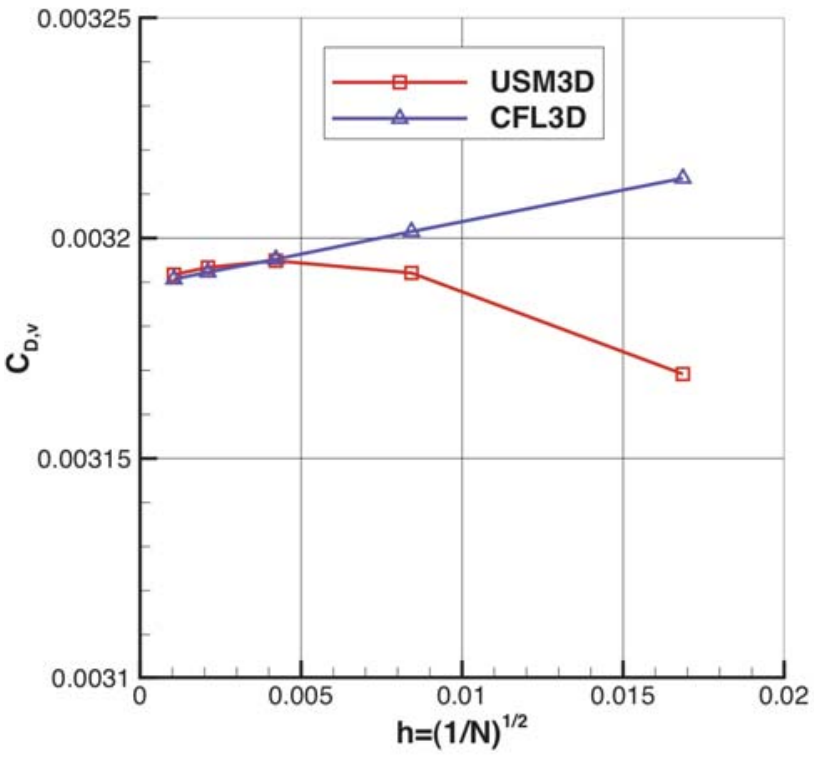

(b) integrated viscous drag coefficient

Figure 10. Grid convergence of $2 \mathrm{D}$ bump-in-channel skin friction and viscous drag coefficients computed using SA model on a sequence of five hexahedral grids. $M_{\infty}=0.2, \operatorname{Re}_{L}=3 \times 10^{6}(L=1)$. 


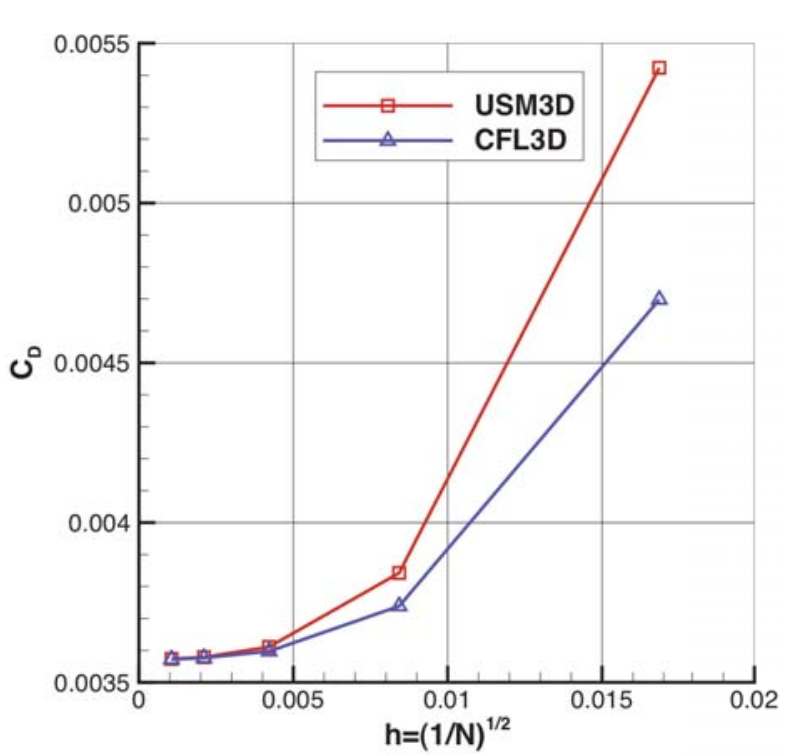

(a) integrated total drag coefficient

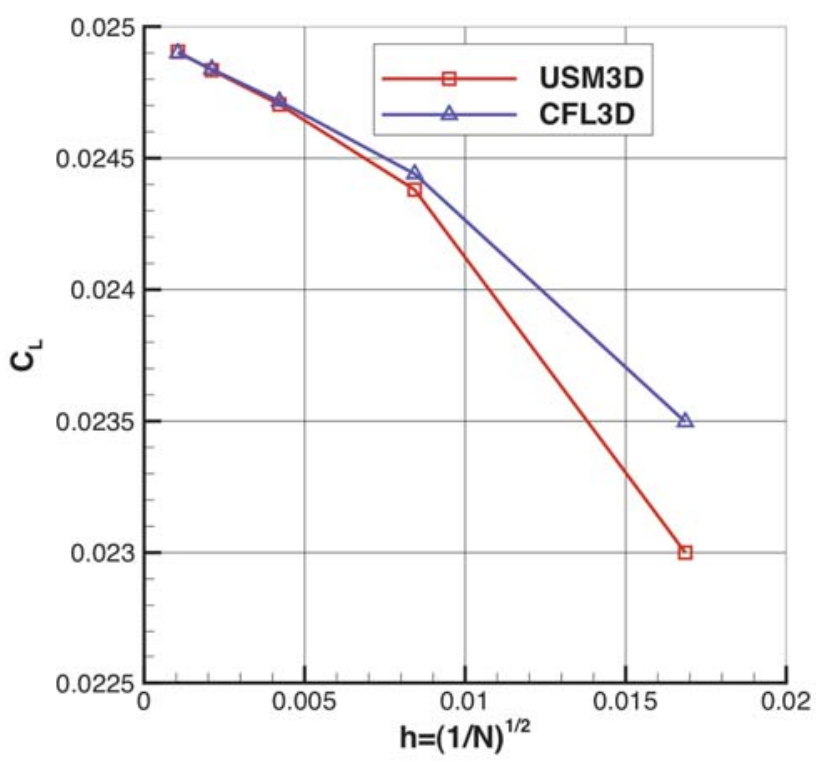

(b) integrated lift coefficient

Figure 11. Grid convergence of 2D bump-in-channel total drag and lift coefficients computed using SA model on a sequence of five hexahedral grids. $M_{\infty}=0.2, \operatorname{Re}_{L}=3 \times 10^{6}(L=1)$.

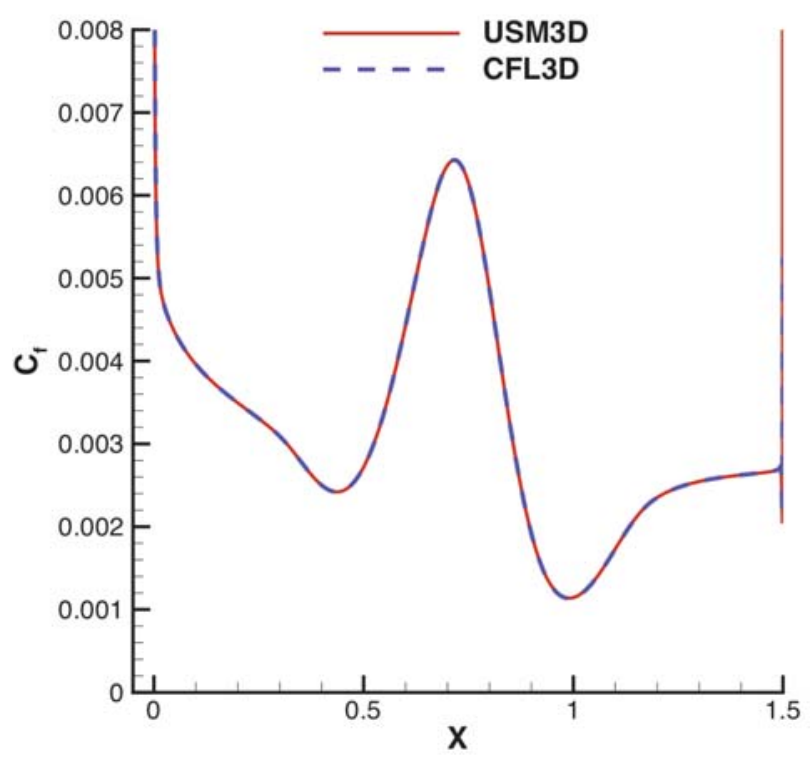

(a) skin friction coefficient

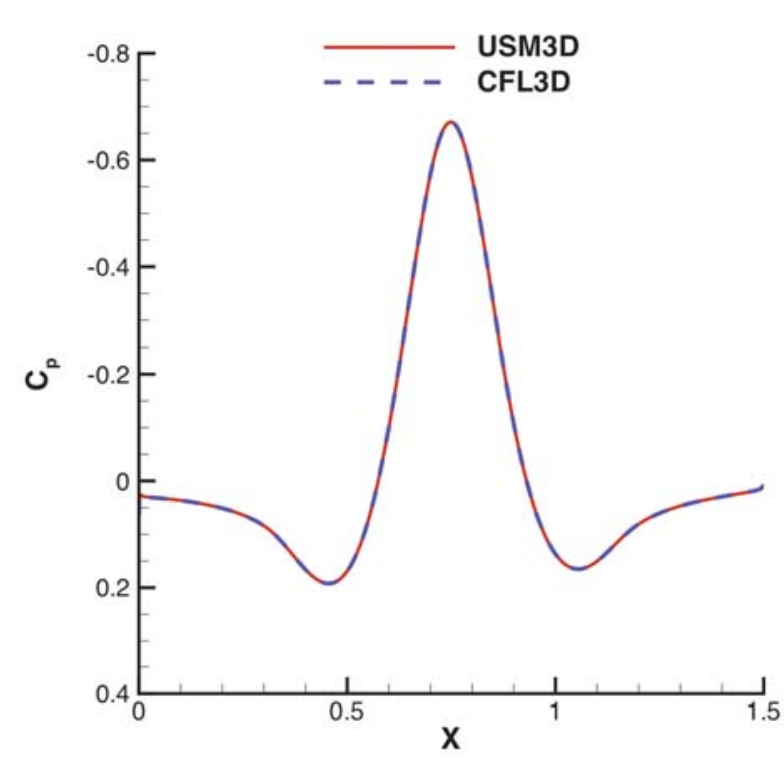

(b) coefficient of surface pressure

Figure 12. Comparison of the streamwise variations of skin friction and surface pressure coefficients computed using SA model on the finest hexahedral grid for the 2D bump-in-channel. $M_{\infty}=0.2, \operatorname{Re}_{L}=3 \times 10^{6}(L=1)$. 


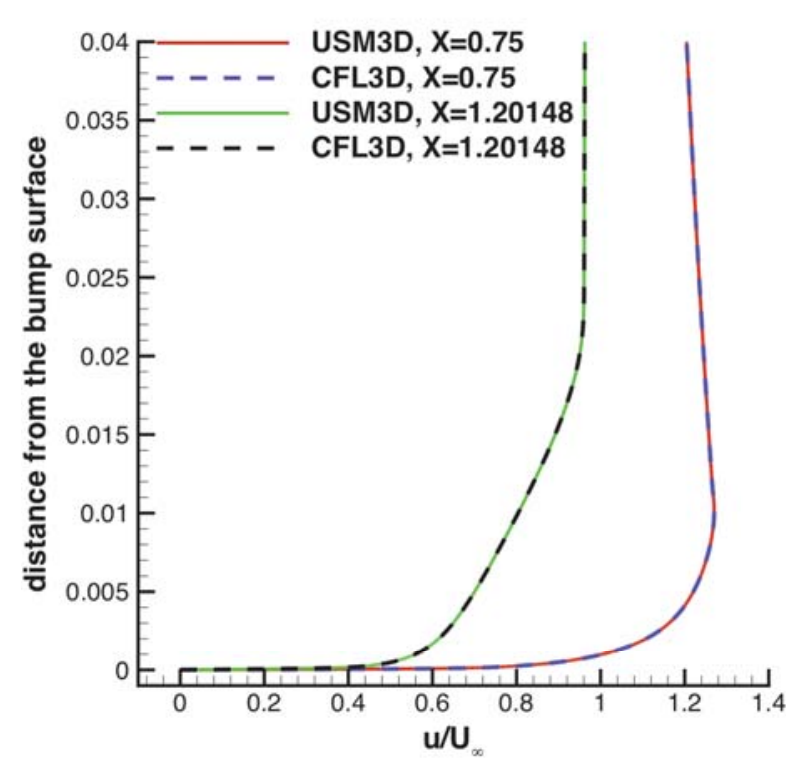

(a) streamwise velocity at two streamwise stations

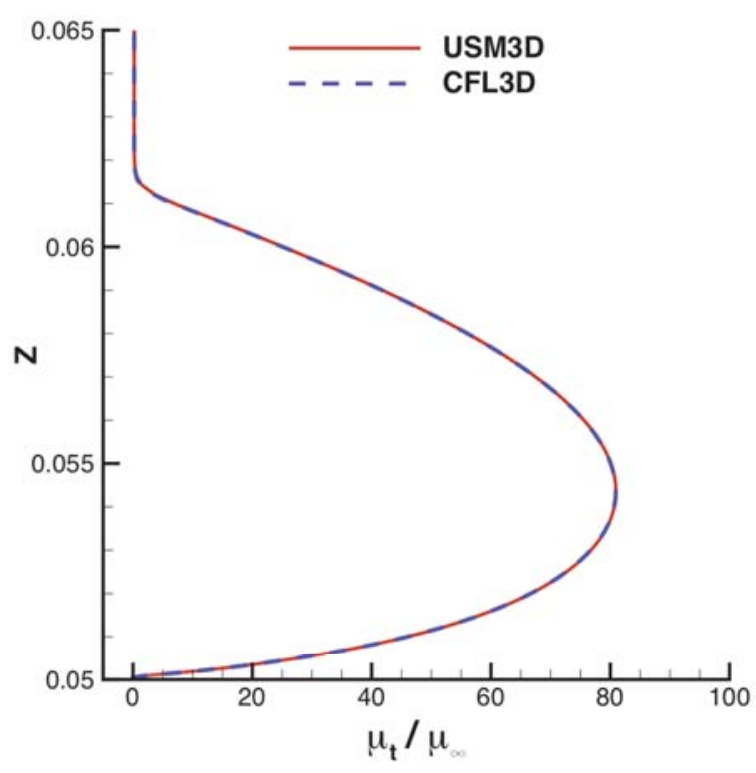

(b) eddy viscosity at $\mathrm{X}=0.97$

Figure 13. Comparison of the normal direction variations of the normalized streamwise velocity and eddy viscosity computed using SA model on the finest hexahedral grid for the $2 \mathrm{D}$ bump-in-channel. $M_{\infty}=0.2, \operatorname{Re}_{\mathrm{L}}=3 \times 10^{6}(\mathrm{~L}=1)$.

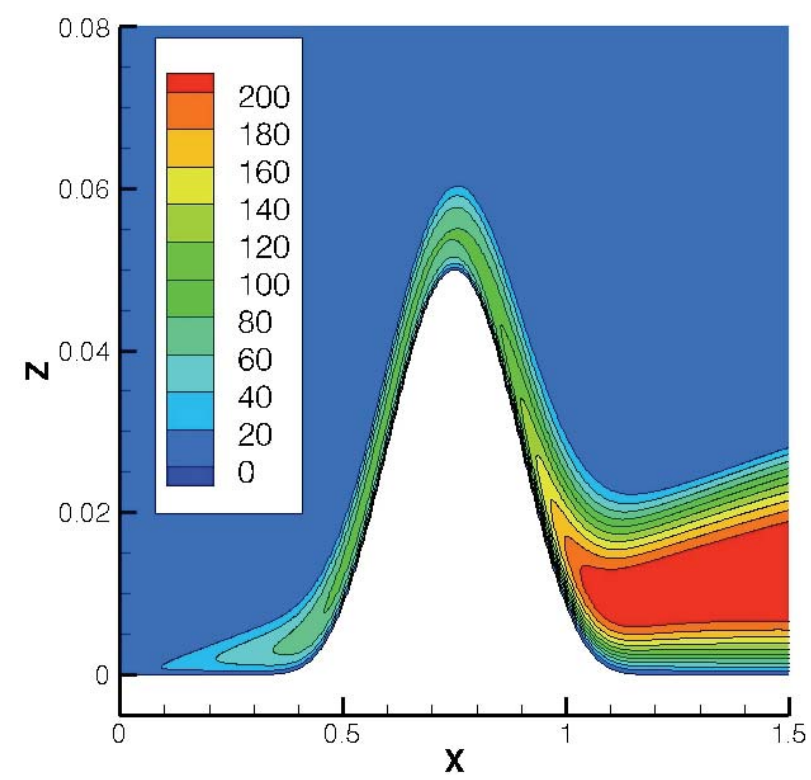

(a) USM3D

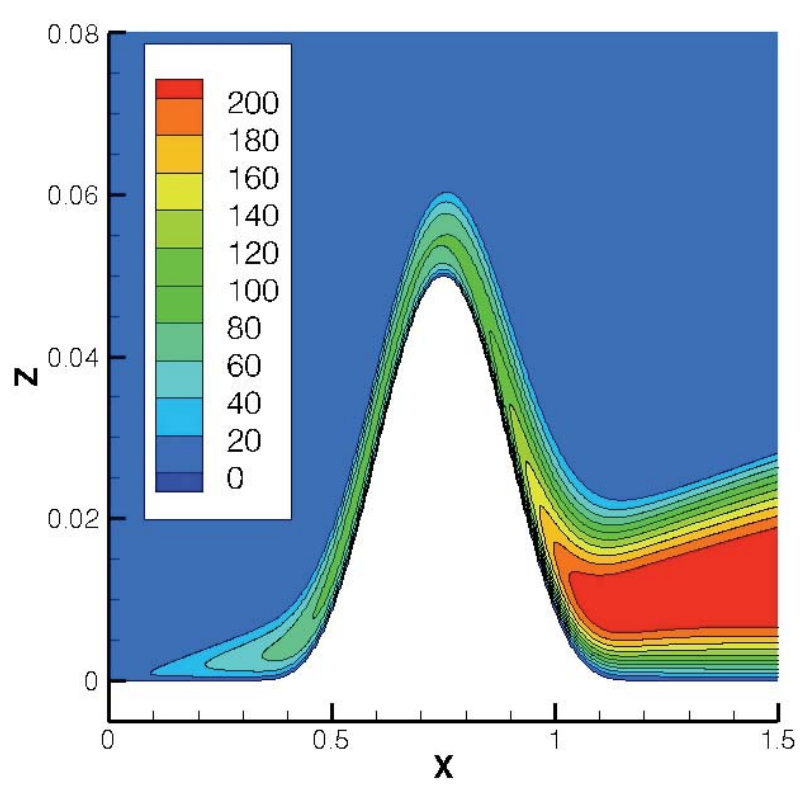

(b) CFL3D

Figure 14. Nearfield normalized eddy viscosity contours from USM3D and CFL3D computed using SA model on the finest hexahedral grid for the 2D bump-in-channel. $M_{\infty}=0.2, \operatorname{Re}_{L}=3 \times 10^{6}(L=1)$. 


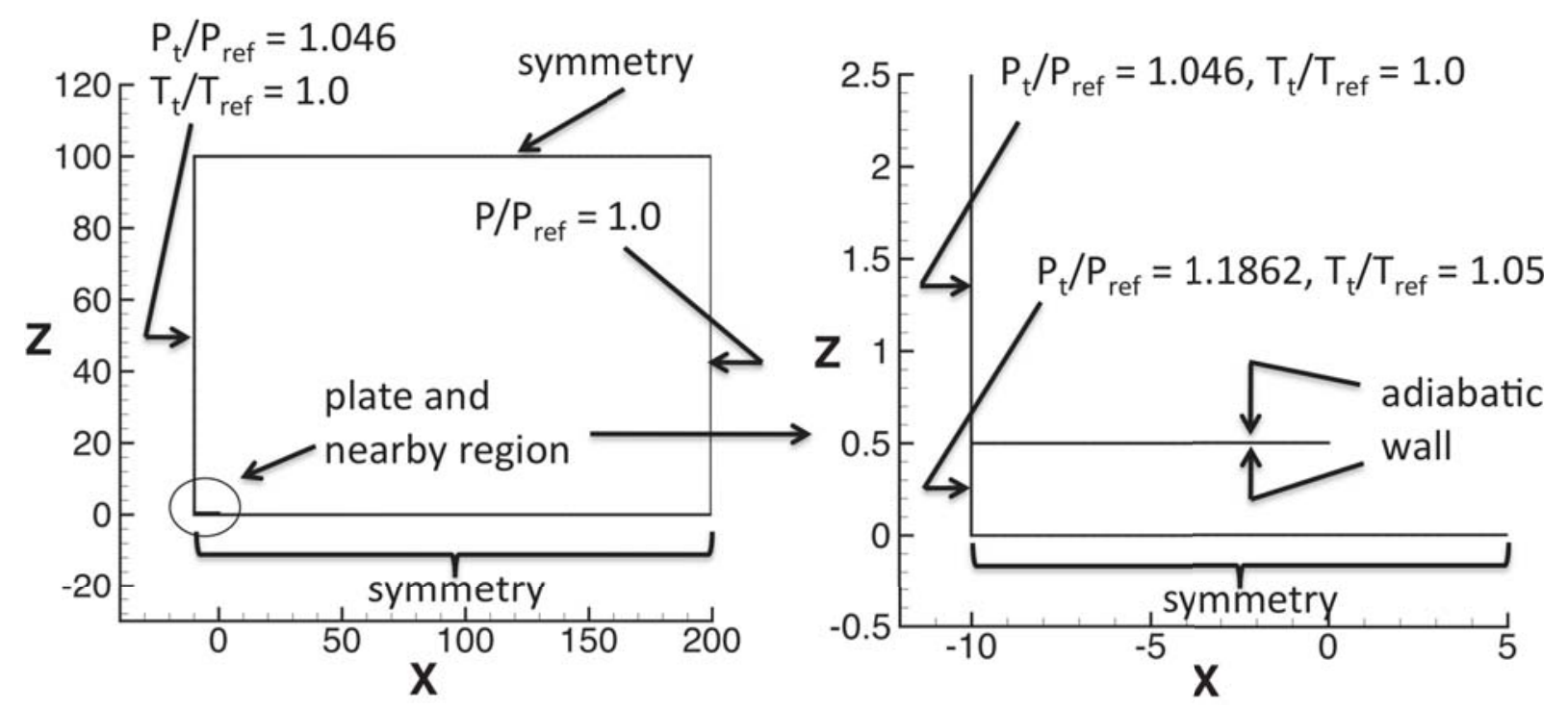

Figure 15. Schematic view of computational domain and various boundary conditions for the 2D planar shear case.

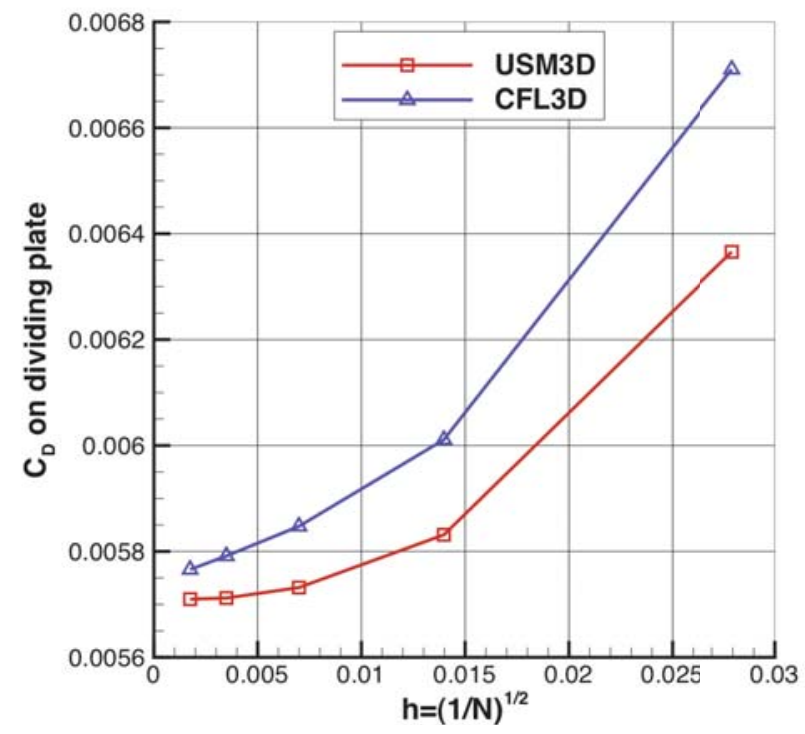

(a) integrated drag on dividing plate

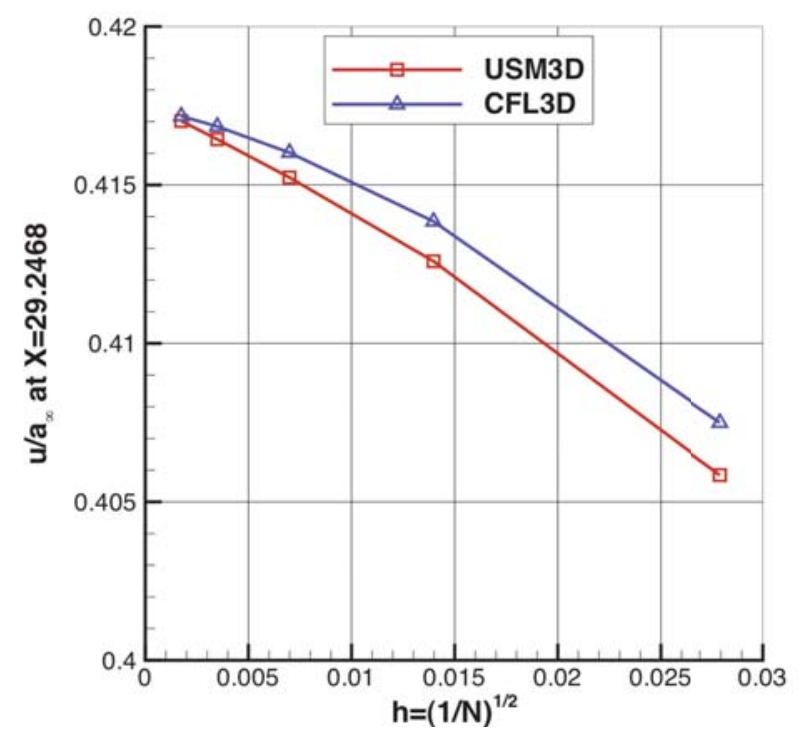

(b) streamwise velocity at $\mathrm{X}=\mathbf{2 9 . 2 4 6 8}$

Figure 16. Grid convergence of drag coefficient and local normalized streamwise velocity for the 2D planar shear case computed using $S A$ model on a sequence of five hexahedral grids. $M_{\infty}=0.5, \operatorname{Re}_{L}=50,000(L=1)$. 


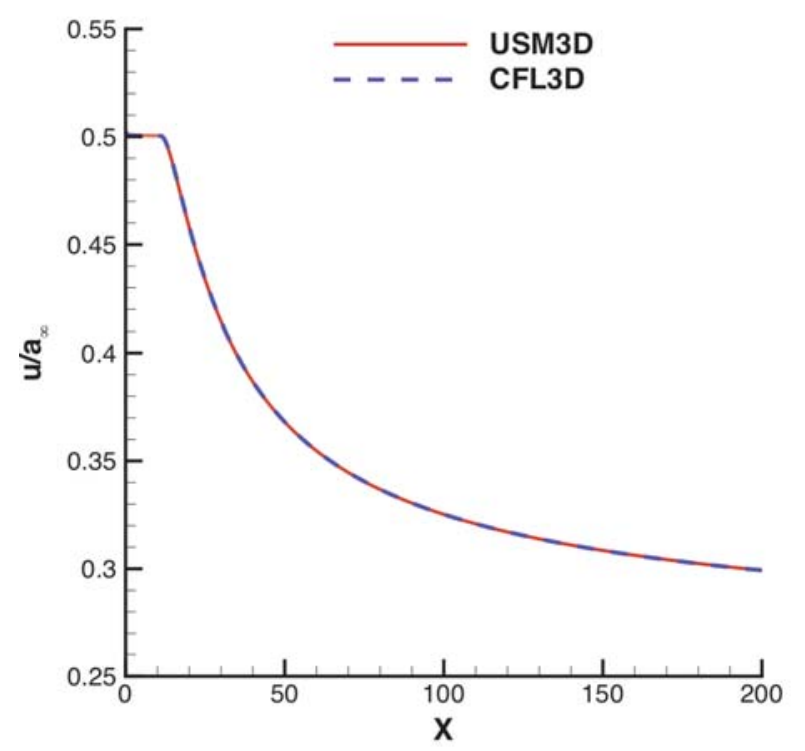

Figure 17. Comparison of the normalized streamwise velocity along $Z=0$ station computed using $S A$ model on the finest hexahedral grid for the $2 \mathrm{D}$ planar shear case. $M_{\infty}=0.5, \mathrm{Re}_{\mathrm{L}}=\mathbf{5 0 , 0 0 0}(\mathrm{L}=1)$.

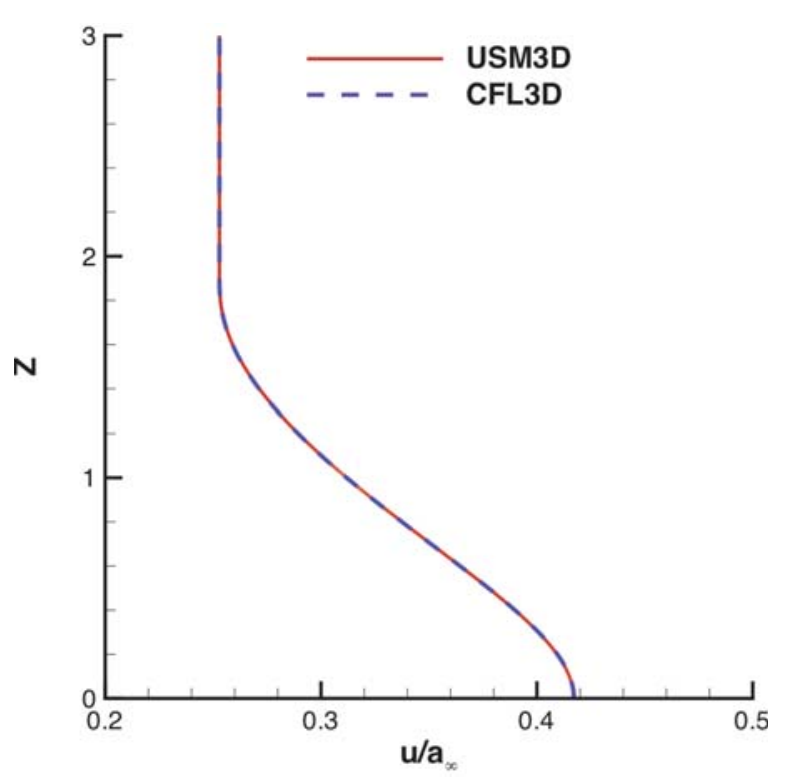

(a) streamwise velocity at $\mathrm{X}=\mathbf{2 9 . 2 4 6 8}$

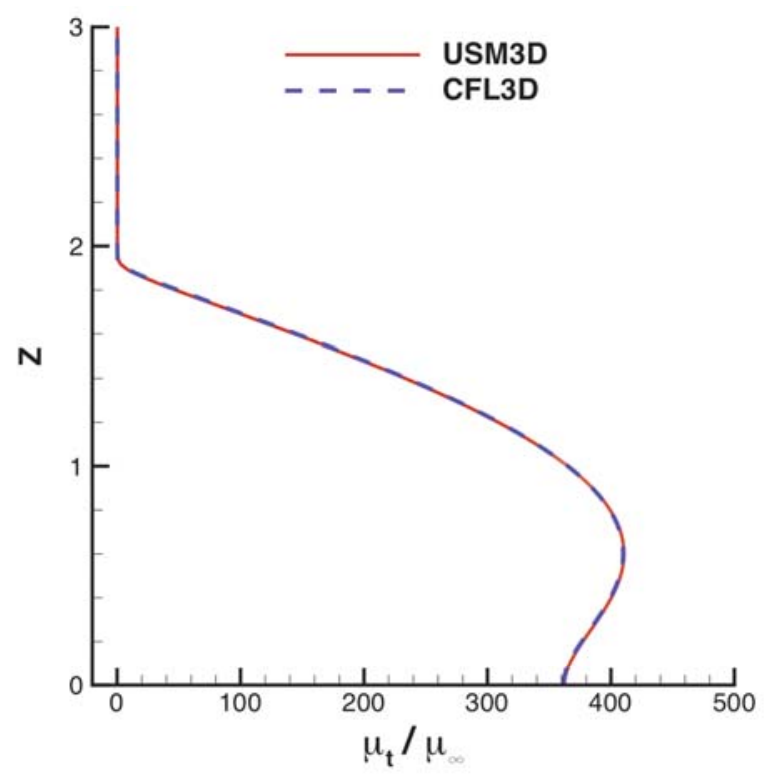

(b) eddy viscosity at $X=29.2468$

Figure 18. Comparison of the normal direction variations of the normalized streamwise velocity and eddy viscosity computed using SA model on the finest hexahedral grid for the $2 \mathrm{D}$ planar shear case. $M_{\infty}=0.5, \operatorname{Re}_{L}=50,000(L=1)$. 


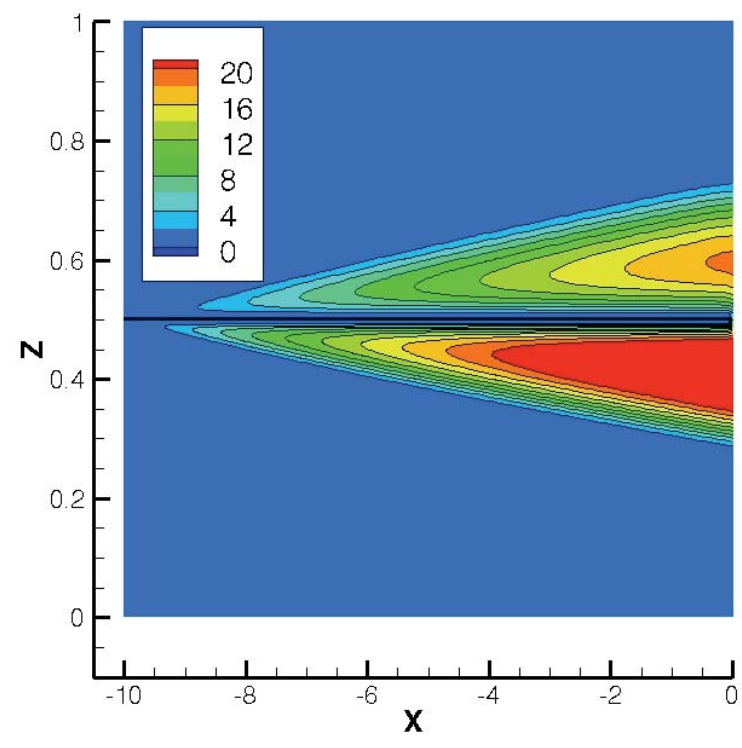

(a) USM3D

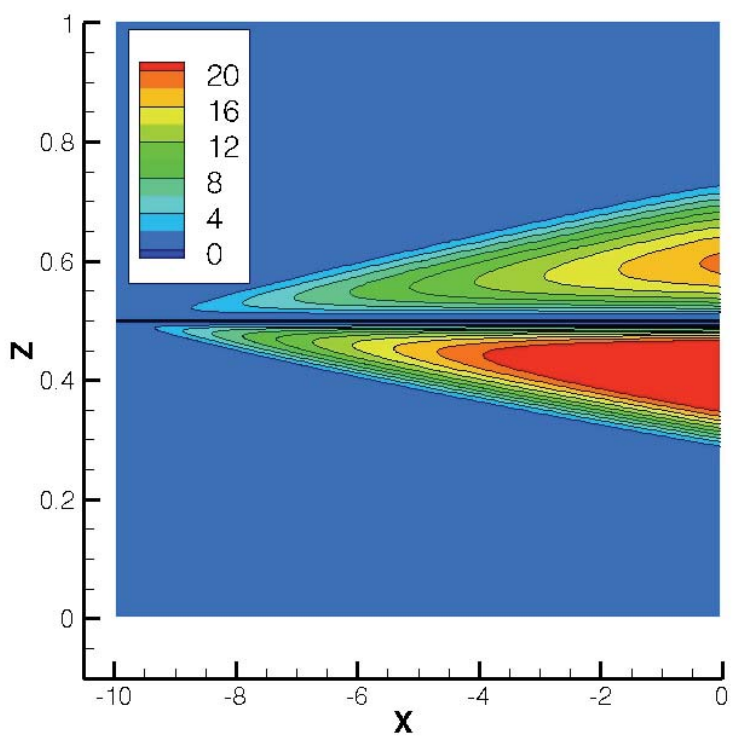

(b) CFL3D

Figure 19. Nearfield normalized eddy viscosity contours from USM3D and CFL3D computed using SA model on the finest hexahedral grid for the 2D planar shear case. $M_{\infty}=0.5, R_{L}=50,000(L=1)$.

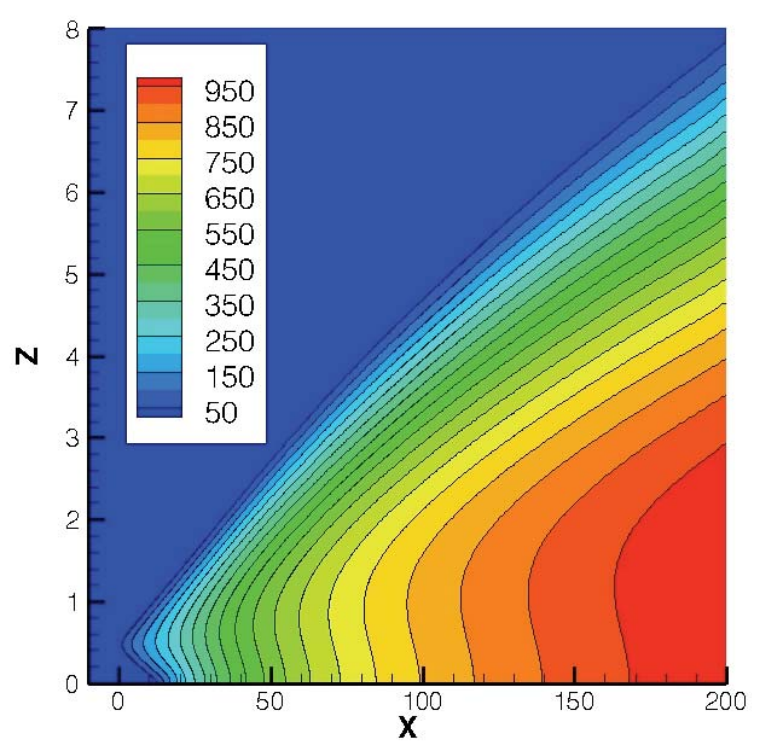

(a) USM3D

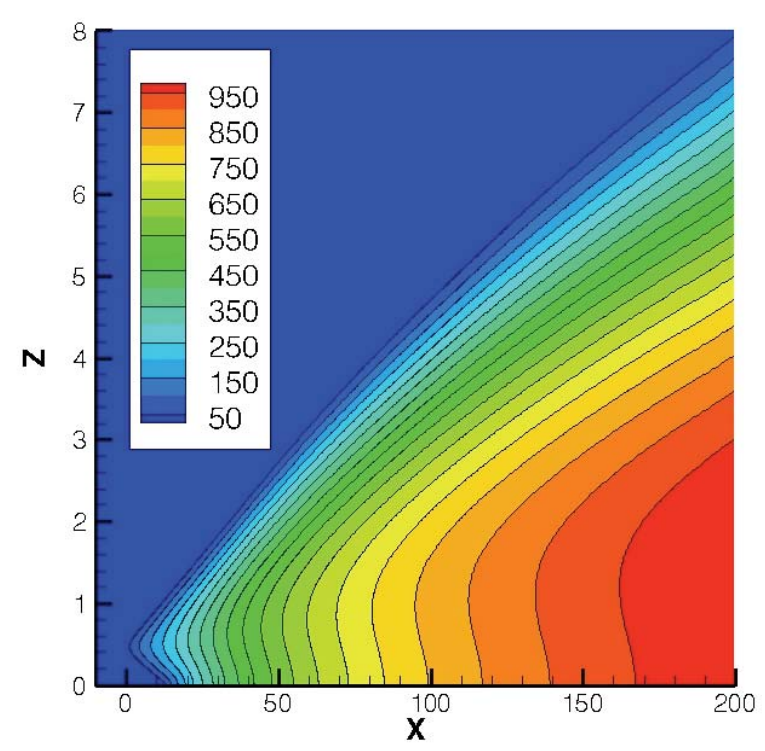

(b) CFL3D

Figure 20. Farfield normalized eddy viscosity contours from USM3D and CFL3D computed using SA model on the finest hexahedral grid for the 2D planar shear case. $M_{\infty}=0.5, \operatorname{Re}_{L}=50,000(L=1)$. 


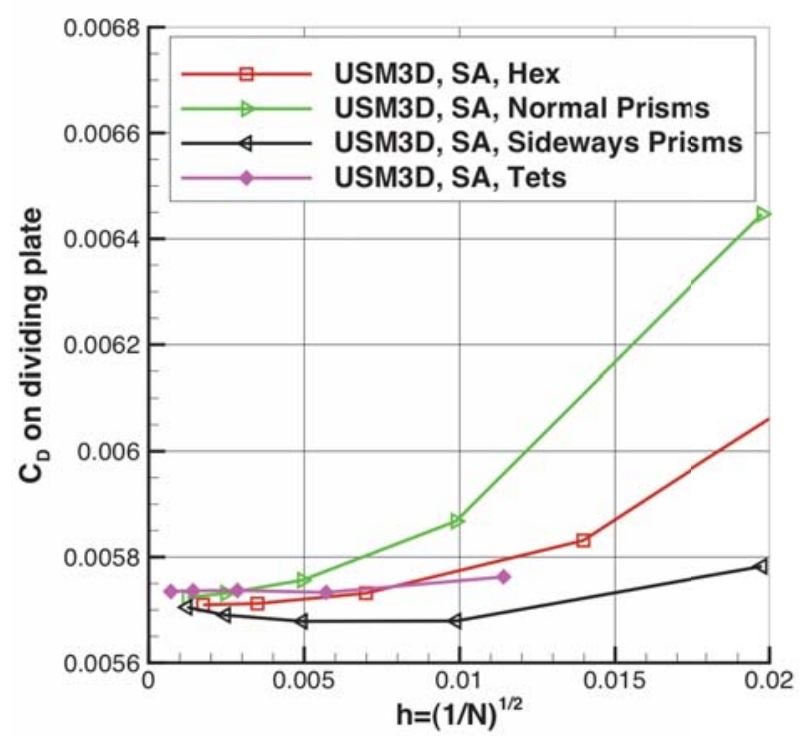

(a) integrated drag on dividing plate

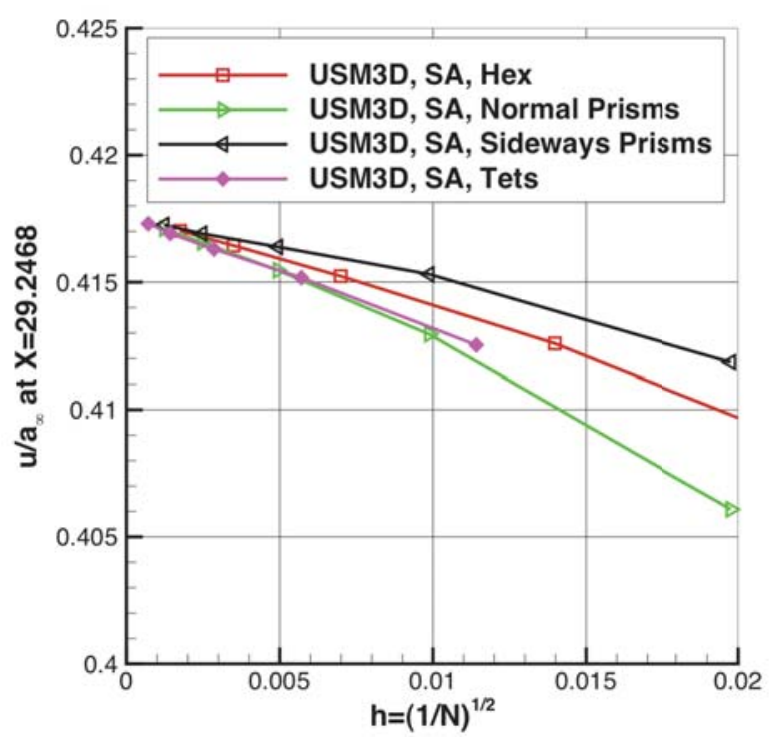

(b) streamwise velocity at $\mathrm{X}=\mathbf{2 9 . 2 4 6 8}$

Figure 21. Grid convergence of drag coefficient and local normalized streamwise velocity for the 2D planar shear case computed using $S A$ model on a sequence of five grids of four different topologies. $M_{\infty}=0.5, \operatorname{Re}_{L}=50,000(L=1)$.

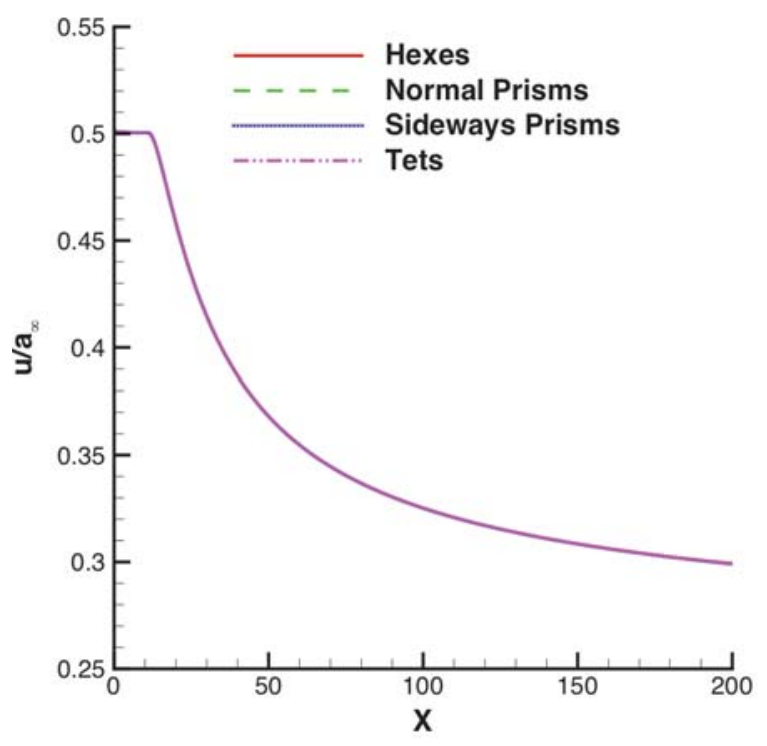

Figure 22. Comparison of the normalized streamwise velocity along $Z=0$ station computed using $S A$ model on the finest grid from four different topologies for the 2D planar shear case. $M_{\infty}=0.5, \operatorname{Re}_{L}=50,000(L=1)$. 


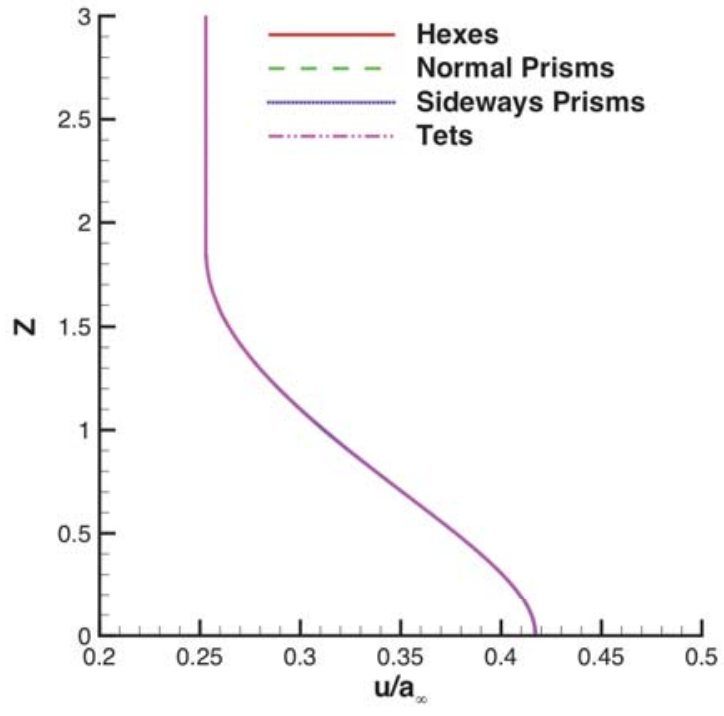

(a) streamwise velocity at $X=29.2468$

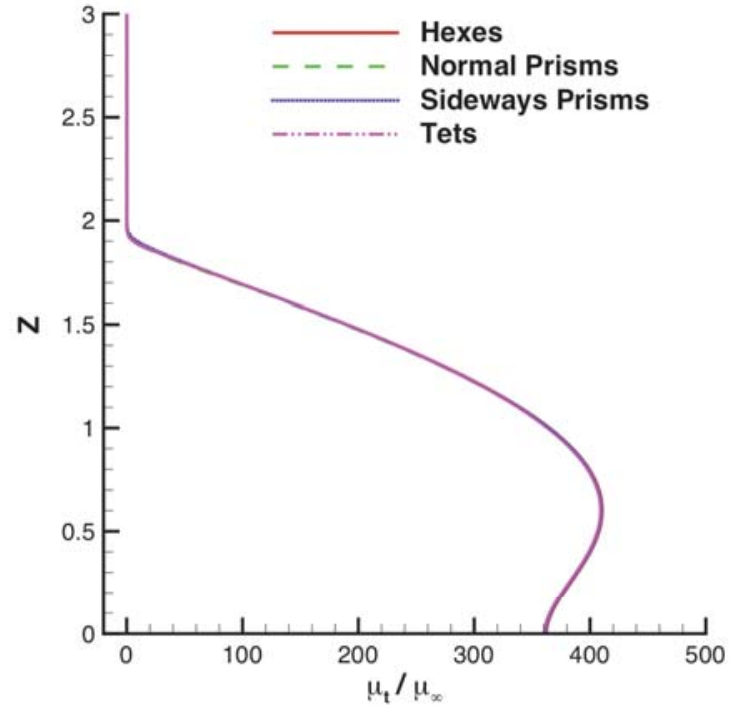

(b) eddy viscosity at $\mathrm{X}=\mathbf{2 9 . 2 4 6 8}$

Figure 23. Comparison of the normal direction variations of the normalized streamwise velocity and eddy viscosity computed using SA model on the finest grid from four different topologies for the $2 D$ planar shear case. $M_{\infty}=0.5$, $\operatorname{Re}_{\mathrm{L}}=\mathbf{5 0 , 0 0 0}(\mathbf{L}=\mathbf{1})$.

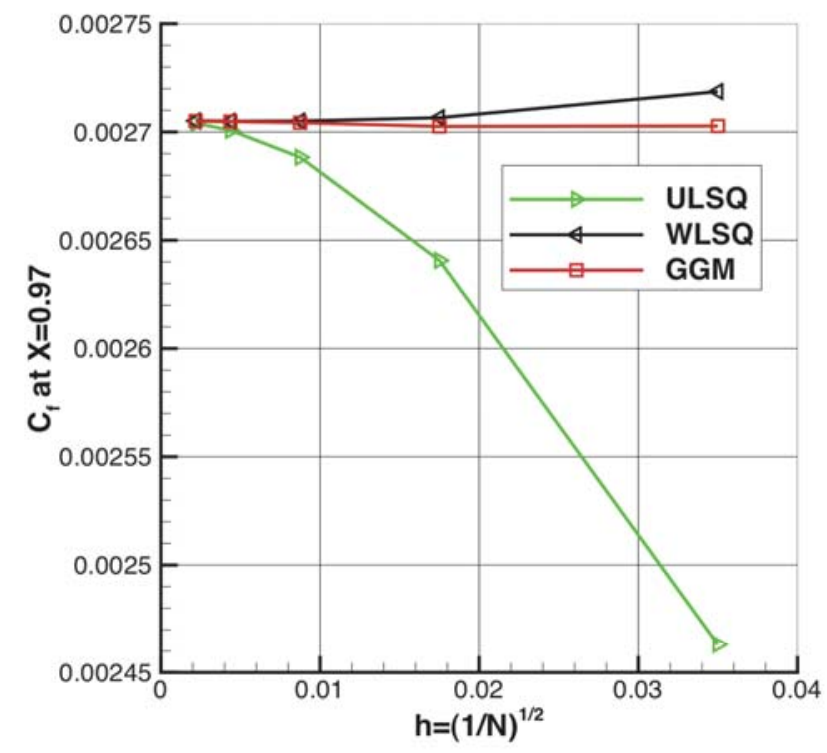

(a) local skin friction at $\mathrm{X}=\mathbf{0 . 9 7}$

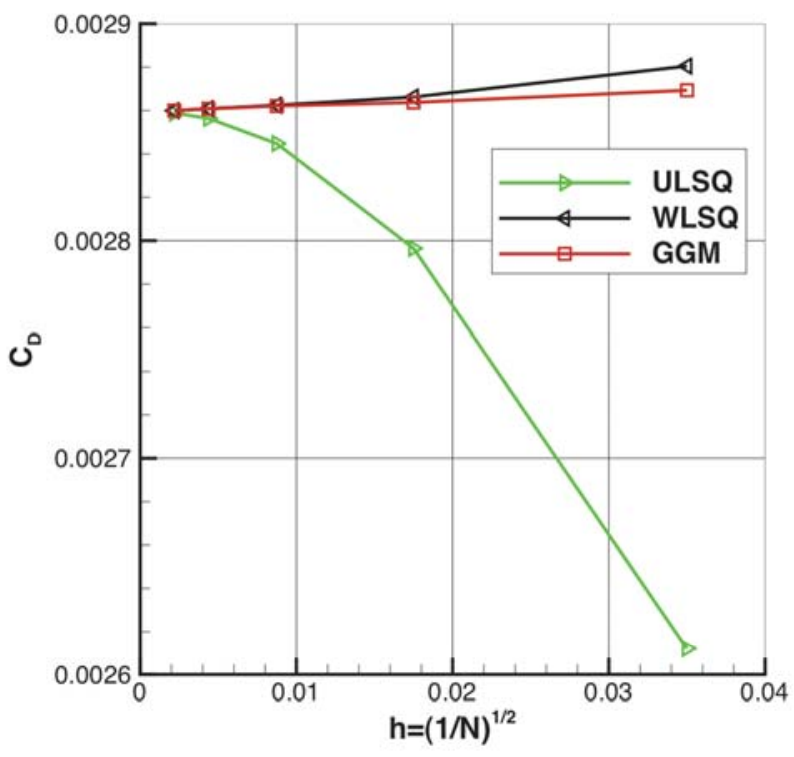

(b) integrated drag coefficient

Figure 24. Grid convergence of skin friction and drag coefficients for the 2D zero pressure gradient flat plate computed using USM3D SA model and various gradient calculation procedures on a sequence of five hexahedral grids. $\mathrm{M}_{\infty}=0.2, \operatorname{Re}_{\mathrm{L}}=5 \times 10^{6}(\mathrm{~L}=1)$. 


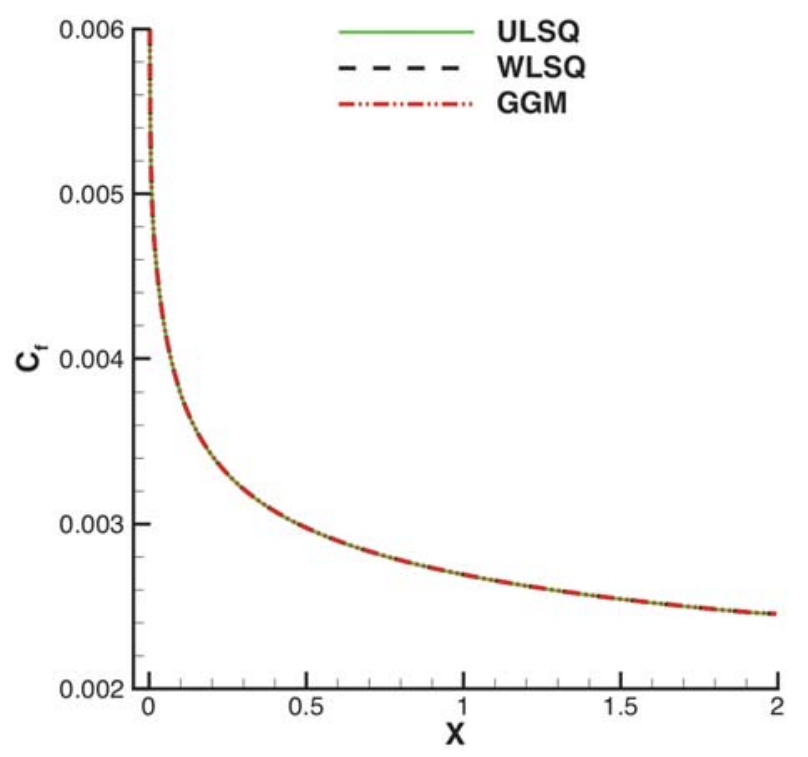

Figure 25. Comparison of the streamwise variations of local skin friction computed using USM3D SA model and various gradient calculation procedures on the finest hexahedral grid for the $2 \mathrm{D}$ zero pressure gradient flat plate. $M_{\infty}=0.2, \operatorname{Re}_{L}=5 \times 10^{6}(\mathrm{~L}=1)$.

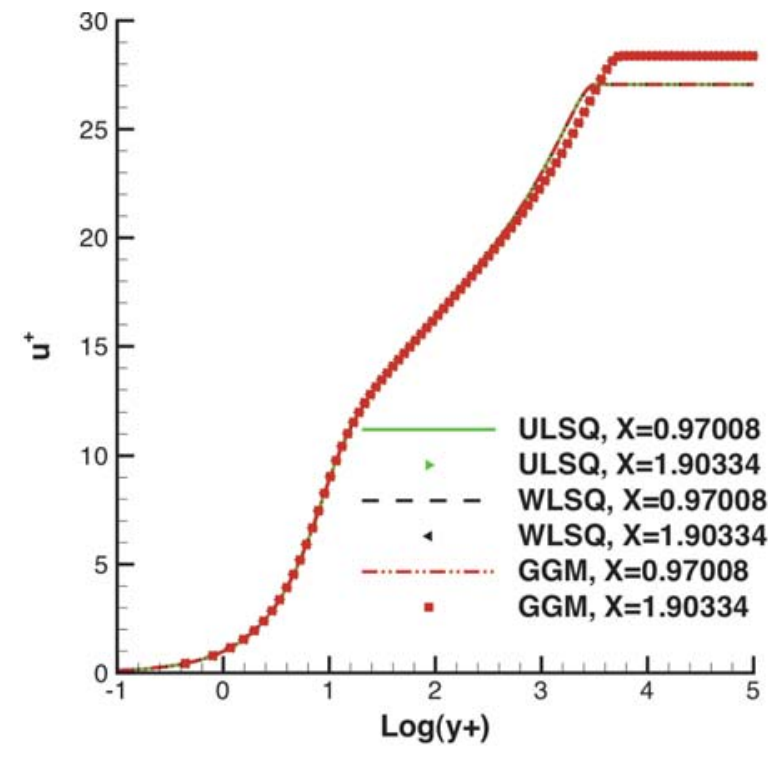

(a) streamwise velocity at two streamwise stations

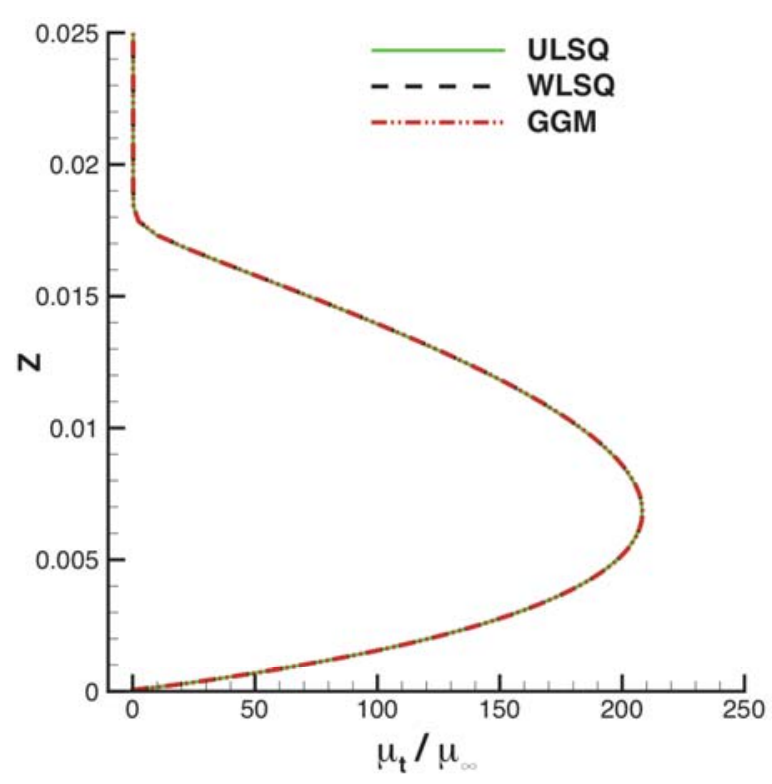

(b) eddy viscosity at $\mathrm{X}=\mathbf{0 . 9 7}$

Figure 26. Comparison of the normal direction variations of streamwise velocity and eddy viscosity computed using USM3D SA model and various gradient calculation procedures on the finest hexahedral grid for the $2 \mathrm{D}$ zero pressure gradient flat plate. $M_{\infty}=0.2, \operatorname{Re}_{L}=5 \times 10^{6}(L=1)$. 